\title{
Detection and risk assessments of multi-pesticides in 1771 cultivated herbal medicines by LC/MS-MS and GC/MS-MS
}

Luo, Lu; Dong, Linlin; Huang, Qin; Ma, Shuangcheng; Fantke, Peter; Li, Jianhui; Jiang, Jingwen; Fitzgerald, Martin; Yang, Jane; Jia, Zhengwei

Total number of authors:

30

Published in:

Chemosphere

Link to article, DOI:

10.1016/j.chemosphere.2020.127477

Publication date:

2021

Document Version

Peer reviewed version

Link back to DTU Orbit

Citation $(A P A)$ :

Luo, L., Dong, L., Huang, Q., Ma, S., Fantke, P., Li, J., Jiang, J., Fitzgerald, M., Yang, J., Jia, Z., Zhang, J., Wang, H., Dai, Y., Zhu, G., Xing, Z., Liang, Y., Li, M., Wei, G., Song, J., ... Chen, S. (2021). Detection and risk assessments of multi-pesticides in 1771 cultivated herbal medicines by LC/MS-MS and GC/MS-MS. Chemosphere, 262, [127477]. https://doi.org/10.1016/j.chemosphere.2020.127477

\section{General rights}

Copyright and moral rights for the publications made accessible in the public portal are retained by the authors and/or other copyright owners and it is a condition of accessing publications that users recognise and abide by the legal requirements associated with these rights.

- Users may download and print one copy of any publication from the public portal for the purpose of private study or research.

- You may not further distribute the material or use it for any profit-making activity or commercial gain

- You may freely distribute the URL identifying the publication in the public portal 


\section{Journal Pre-proof}

Detection and risk assessments of multi-pesticides in 1771 cultivated herbal medicines by LC/MS-MS and GC/MS-MS

Lu Luo, Linlin Dong, Qin Huang, Shuangcheng Ma, Peter Fantke, Jianhui Li, Jingwen Jiang, Martin Fitzgerald, Jane Yang, Zhengwei Jia, Jiqing Zhang, Haifeng Wang, Yuntao Dai, Guangwei Zhu, Zhihan Xing, Yichuan Liang, Mengzhi Li, Guangfei Wei, Jingyuan Song, Jianhe Wei, Cheng Peng, Han Zhang, Wei Zhang, Shumei Wang, Kaito Mizuno, Alarcon Arauco Gian Marco, Lan Wu, Jiang Xu, Chao Xiong, Shilin Chen

PII: S0045-6535(20)31671-4

DOI: https://doi.org/10.1016/j.chemosphere.2020.127477

Reference: CHEM 127477

To appear in: ECSN

Received Date: 16 April 2020

Revised Date: 20 June 2020

Accepted Date: 20 June 2020

Please cite this article as: Luo, L., Dong, L., Huang, Q., Ma, S., Fantke, P., Li, J., Jiang, J., Fitzgerald, M., Yang, J., Jia, Z., Zhang, J., Wang, H., Dai, Y., Zhu, G., Xing, Z., Liang, Y., Li, M., Wei, G., Song, J., Wei, J., Peng, C., Zhang, H., Zhang, W., Wang, S., Mizuno, K., Gian Marco, A.A., Wu, L., Xu, J., Xiong, C., Chen, S., Detection and risk assessments of multi-pesticides in 1771 cultivated herbal medicines by LC/MS-MS and GC/MS-MS, Chemosphere, https://doi.org/10.1016/j.chemosphere.2020.127477.

This is a PDF file of an article that has undergone enhancements after acceptance, such as the addition of a cover page and metadata, and formatting for readability, but it is not yet the definitive version of record. This version will undergo additional copyediting, typesetting and review before it is published in its final form, but we are providing this version to give early visibility of the article. Please note that, during the production process, errors may be discovered which could affect the content, and all legal disclaimers that apply to the journal pertain.

(C) 2020 Elsevier Ltd. All rights reserved. 
Shilin Chen: conceptualization, supervision, project administration and funding acquisition.

Lu Luo: Sample preparation, information collection, data analysis, risk assessments and writing- original draft preparation, figure and creation, reviewing and editing, Linlin Dong: Methodology.

Guangwei Zhu, Jingyuan Song, Jianhe Wei, Cheng Peng, Han Zhang, and Wwei

Zhang: sample collection.

ShumeiWang, and Lan Wu: sample preparations.

Jianhui Li, JaneYang, and Zhengwei Jia: chemical analysis.

Qin Huang: information collection and data analysis

Zhihan Xing: information collection.

Jiqing Zhang, Yichuan Liang, Guangfei Wei, Mengzhi Li, Haifeng Wang, Yuntao

Dai, Chao Xiong, and Shuangcheng Ma: data analysis.

Jingwen Jiang, Alarcon Arauco Gian Marco, and Jiang Xu: figure creation.

Peter Fantke: comparative patient health impact assessment.

Martin Fitzgerald: solution strategy, reviewing and editing.

Jiaxing Liu: reviewing and editing. 


\section{Detection and risk assessments of multi-pesticides in 1771 cultivated herbal}

\section{medicines by LC/MS-MS and GC/MS-MS}

Authors: Lu Luo ${ }^{1 \dagger}$, Linlin Dong ${ }^{1 \dagger}$, Qin Huang ${ }^{1}$, Shuangcheng $\mathrm{Ma}^{2}$, Peter Fantke ${ }^{3}$, Jianhui $\mathrm{Li}^{4}$, Jingwen Jiang $^{5}$, Martin Fitzgerald ${ }^{6}$, Jane Yang ${ }^{4}$, Zhengwei Jia ${ }^{4}$, Jiqing Zhang ${ }^{1}$, Haifeng Wang ${ }^{1}$, Yuntao Dai ${ }^{1}$, Guangwei Zhu ${ }^{1}$, Zhihan Xing ${ }^{7}$, Yichuan Liang ${ }^{1}$, Mengzhi Li $^{1}$, Guangfei Wei ${ }^{1}$, Jingyuan Song ${ }^{8}$, Jianhe Wei $^{8}$, Cheng Peng ${ }^{9}$, Han Zhang ${ }^{10}$, Wei Zhang ${ }^{11}$, Shumei Wang ${ }^{1}$, Kaito Mizuno ${ }^{12}$, Alarcon Arauco Gian

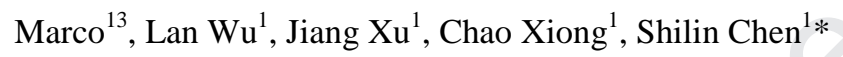

\section{Affiliations:}

${ }^{1}$ Institute of Chinese Materia Medica, China Academy of Chinese Medical Sciences, Beijing 100700, PR China.

${ }^{2}$ National Institutes for Food and Drug Control, Beijing 100050, PR China

${ }^{3}$ Quantitative Sustainability Assessment, Department of Technology, Management and Economics,

Technical University of Denmark, Produktionstorvet 424, 2800 Kgs. Lyngby, Denmark.

${ }^{4}$ Waters Technologies Shanghai Limited, Block 13, City of Elite, 1000 Jinhai Road, Pu Dong New District, Shanghai 201206, PR China. 
${ }^{5}$ West China Biomedical Big Data Center, West China Hospital, Sichuan University, Chengdu 610041,

PR China.

${ }^{6}$ Department of Life Sciences, University of Westminster, 115 New Cavendish Street, W1W 6UW,

London, UK.

${ }^{7}$ College of Science and Mathematics, University of Massachusetts Boston, 100 William T. Morrissey

Blvd. Boston, MA 02125-3393, USA.

${ }^{8}$ Institute of Medicinal Plant Development, Chinese Academy of Medical Sciences, Peking Union

Medical College, Beijing 100193, PR China.

${ }^{9}$ Pharmacy College, Chengdu University of Traditional Chinese Medicine, Chengdu 611137, Sichuan,

PR China.

${ }^{10}$ Institute of Traditional Chinese Medicine, Tianjin University of Traditional Chinese Medicine, Tianjin

301617, PR China.

${ }^{11}$ Pharmacogenetics Research Institute, Institute of Clinical Pharmacology, Central South University,

Changsha 410008, Hunan, PR China.

${ }^{12}$ Suzuka University of Medical Science, 1001-1, Kishioka, Suzuka, 510-0293, Japan. 
${ }^{13}$ Intelligence of Science and Technology, School of Automation and Electrical Engineering, University of Science and Technology, Beijing 100083, PR China.

*Correspondence to: Prof Shilin Chen, Institute of Chinese Materia Medica, China Academy of Chinese Medical Sciences, Beijing 100700, China, slchen@icmm.ac.cn.

\section{CRediT authorship contribution statement}

Shilin Chen: conceptualization, supervision, project administration and funding acquisition. Lu Luo: Sample preparation, information collection, data analysis, risk assessments and writing- original draft preparation, figure and creation, reviewing and editing. Linlin Dong: Methodology and reviewing. Guangwei Zhu, Jingyuan Song, Jianhe Wei, Cheng Peng, Han Zhang, and Wwei Zhang: sample collection. ShumeiWang, and Lan Wu: sample preparations. Jianhui Li, JaneYang, and Zhengwei Jia: chemical analysis. Qin Huang: information collection and data analysis. Zhihan Xing: information collection. Jiqing Zhang, Yichuan Liang, Guangfei Wei, Mengzhi Li, Haifeng Wang, Yuntao Dai, Chao Xiong, and Shuangcheng Ma: data analysis. Jingwen Jiang, Alarcon Arauco Gian Marco, and Jiang Xu: figure creation. Peter Fantke: comparative patient health impact assessment. Martin Fitzgerald: solution strategy, reviewing and editing. Jiaxing Liu: reviewing and editing. 


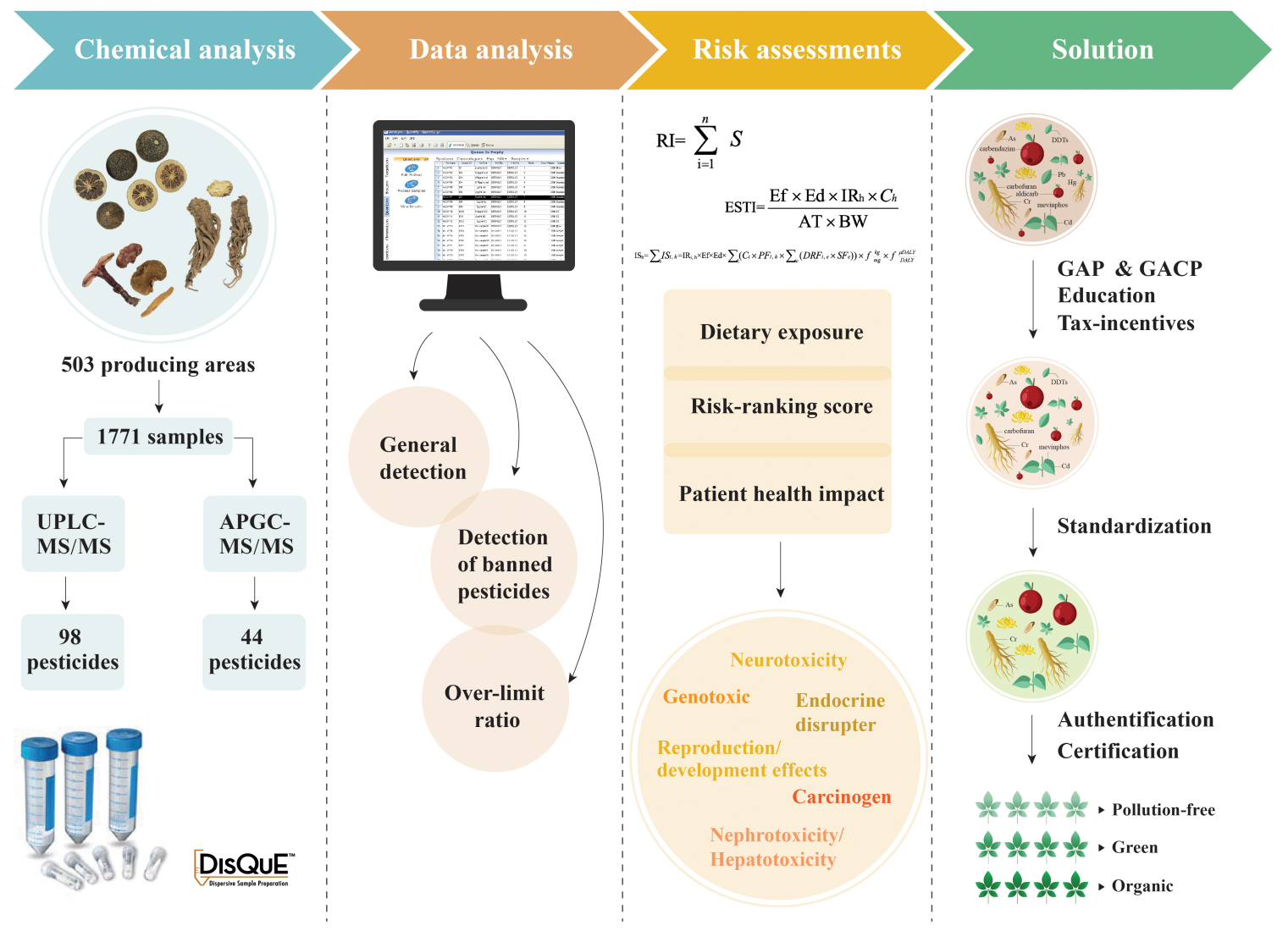


1 Detection and risk assessments of multi-pesticides in 1771 cultivated herbal

3

4 medicines by LC/MS-MS and GC/MS-MS

\section{Abstract}

Focus on the safety of herbal medicines has mainly been directed towards the presence of intrinsic toxicity, as found in the cases of renal and hepatic dysfunction caused by aristolochic acids. However, contamination from extrinsic hazards may impart an even greater reduction in their safety and efficacy.

This study reveals that pesticides were present in the majority (88\%) of a comprehensive cross-section $(n=1771)$ of herbal medicine samples. Alarmingly, more than half $(59 \%)$ contained pesticides over the European Pharmacopoeia (EP) limit, and $43 \%$ of them contained 35 varieties of banned, extremely toxic pesticides, eight of which were detected at levels over 500 times higher than the default Maximum Residue Limit (MRL). DDTs, carbofuran, and mevinphos were confirmed as being among the most risk-inducing pesticides by three different risk assessment methods, reported to produce carcinogenic, genotoxic, reproductive, and developmental effects, in addition to carrying nephrotoxicity and hepatotoxicity. In light of these findings, and withstanding that extrinsic hazards can be controlled unlike intrinsic toxicity, the authors here strongly recommend the application of herbal medicine quality-control measures and solutions to safeguard against a neglected but certainly potentially serious health risk posed to the majority of the global population that consumes herbal medicines.

\section{Key words}

Herbal medicines; pesticide; extrinsic contamination; risk assessment; public health. 


\section{Introduction}

Herbal medicines (HM) play both important medicinal and economic roles internationally, with $80 \%$ of the world's population choosing traditional medicines to meet their healthcare needs (WHO, 2013), and the global HM market reaching an estimated \$107 billion in 2017 (Sma, 2016). These figures are expected to rise with the rapidly expanding globalization and commercialization of HM. Multiple large-scale epidemiological studies claim that the higher occurrence of nephropathy found in Asia compared to other continents is inherently due to more frequent exposure to HM in this region. (Yang et al., 2014; Broe et al., 2012; Lord et al., 1999; Nortier et al., 2000; Debelle et al., 2008; Ng et al., 2017) Attention to the cause of adverse effects from HM has focused on intrinsic toxicity, as seen in the highly reported cases of hepatocellular carcinoma (Ng et al., 2017) and urothelial malignancies. (Broe et al., 2012; Lord et al., 1999; Nortier et al., 2000; Debelle et al., 2008) However, the extrinsic aspects of toxicity, pesticides in particular, are often neglected despite these being persistent, bioaccumulative, and toxic. These extrinsic toxins are ranked highly amongst suspected carcinogens and endocrine disruptors (Hajšlová et al., 2003), such as p,p'-DDT, aldicarb, and mevinphos. They possess a more serious potential hazard than those of intrinsic nature.

Commercial cultivation of HM has gained ground within an economic climate of sharply rising demand and associated profitability on a global scale (Singh et al., 2018). China is the world's largest producer and supplier of the herbal industry, proving itself the epicenter of the trading of HMs, with an annual production output of approximately over $\$ 200$ billion, which is expected to rise to $\$ 5$ trillion by 2050 (Tripathy et al., 2015). The increasing demand requires more "intensive" 
45

production of HMs leading to higher levels of development in techniques to optimize and maximize profits. The need to protect them from plagues and pests, increase yield and shelf life, reduce post-harvest and storage losses resulted in exponential use of pesticides over the last 50 years (Adusei-Mensah et al., 2018). Concerns were raised for the safety of HM application affecting its commercialization (Singh et al., 2018) regarding contamination with pesticides, microbial contaminants, heavy metals, aflatoxins, chemical toxins, mycotoxins, polycyclic aromatic hydrocarbons (PAHs) and fumigants (Pytlakowska et al., 2012). Particularly, pesticide residues in herbal products may accumulate due to agricultural practices, such as spraying, soil treatment, cultivation in contaminated soil, use of contaminated water sources. These contaminants can also accumulate during the cultivation, storage, and processing of herbs (Tripathy et al., 2015).

The increasing use and the abusive and disordered employment of pesticides will certainly bring a broad spectrum of adverse effects with risks and damages to public health, farm animals, economy, and the alteration of the local environment (Zuin et al., 2000). Highly hazardous pesticides, although banned or strictly controlled, are still heavily promoted and widely applied in HM cultivation, especially in developing nations (Tripathy et al., 2015). Exposure to these contaminants may have cumulative, additive, or synergistic effects on human health (Adusei-Mensah et al., 2018). These contaminants can potentially interfere with the endocrine system, causing endocrine and metabolic disruption, presenting immune-suppressive, carcinogenic, and teratogenic effects (Pytlakowska et al., 2012). They may cause irreversible organ toxicity, fetotoxicity, embryotoxicity, or immunotoxicological effects (Chou et al., 2019) and reproductive issues such as infertility and birth defects. They may also affect and damage the nervous system, causing neurodegenerative and 
67

hepatorenal disorders (Garcia et al., 2012; Adams et al., 2018). Epidemiological studies have associated pesticide contamination with the occurrence of multiple server disorders including cancer, sarcomas, leukemia, non-Hodgkin lymphoma, and myeloma (Garcia et al., 2012). There have been various reports regarding the presence of pesticide contaminants in medicinal herbs. Pesticide residues have been detected in many HMs distributed in the market, especially some are categorized as banned, highly hazardous ones, or extremely hazardous ones by the WHO (Singh et al., 2018). In China, of the 313 samples tested, 94.57\% contained pesticide residues (Chou et al., 2019). A study conducted by American researchers has shown that 42 different pesticides were detected in 108 HM samples (36.7\%) (Zuin et al., 2000). In Portugal, 50\% of the market samples contained organochlorine pesticide residues and $53.1 \%$ of samples were detected with pesticides (Silva et al., 2018). Banned pesticides Aldrin, dieldrin, and chlordane in Ghana were also identified in 50\% of the studied herbal preparations (Adusei-Mensah et al., 2018). In India, a total of 40 samples of single crude herbs (Dashmool) were examined and the results indicated that $5 \%$ contain residue of DDT or its metabolites and presence of $\alpha-\mathrm{HCH}$ and $\gamma-\mathrm{HCH}$, and the main constituents of commercial $\mathrm{HCH}$ were detected in 97.5\% samples (Bhanti et al., 2007). However, the sample numbers and pesticide categories were not sufficient enough to cover all the commonly prescribed in the clinic or widely adopted ones in dietary products. A comprehensive study focusing on commonly used HMs with a large sample number and sampling areas is in urgent need.

However, the current depth-of-coverage pesticide risk assessment in HMs still relies on the comparison of toxicity exposure ratios (Silva et al., 2018), which is always limited due to lack of 
89

90

91

92

pesticide indicator or consumption data. To address these limitations and to grasp a general idea of the types and amount of pesticides applied in HMs, the adoption of banned pesticides, a better categorization of the top-ranking pesticides in HMs and the contributing factors of the risks, a multi-residue pesticide method has been developed for the determination of pesticides in HM. Furthermore, better insight into pesticide contamination over large-scale HM cultivation areas with large representative sample populations is necessary to fully evaluate HM safety on a global scale (Abou-Arab et al., 2001). A more comprehensive risk-assessment approach is needed to fully assess health damage from pesticides to provide a suitable foundation on which to build an effective and well-targeted quality-control strategy. The objectives of this study were to: summarize pesticide content observed in a large sample of cultivated HM samples and compare observed levels to corresponding standards; conduct a comprehensive risk assessment of pesticide contamination in HMs and classify the top risk-inducing pesticides and related HMs; and discuss resolutions to control the quality and efficacy of HMs for policymakers.

\section{Methods}

\subsection{Quality assessment rationale}

For detection rate of banned pesticides, we compiled a list of 35 banned pesticides according to collective regulations of the European Union (EU), United States (US) and China. Banned pesticides from EU were found in Regulation or Directives of EU of the European Parliament and of the Council (EC) (EC, 2004; EC, 2004; EC, 2003;EC, 2003; EC, 2003; EC, 2003.), these from the US were collected from the list of banned and severely restricted pesticides in Prior Informed Consent (PIC) 
110 procedure drafted by the U.S. Environmental Protection Agency (EPA) (Buffington et al., 2018;

111 Colorado State University, 2018; USEPA, 2003; Licensing and Regulatory Affairs, 2018), and these

112 banned in China were obtained from the announcements of Ministry of Agriculture and Rural Affairs of

113 China and Chinese Pharmacopoeia (CP, 2015 edition) (Ministry of Agriculture and Rural Affairs, 2018;

114 National Pharmacopoeia Commission, 2015.).

\subsection{Sample collection} detection. (etection.

Pesticide contamination mainly occurs through agricultural practices. Therefore, a survey of HM categories with major HM cultivation areas and a large artificial cultivation scale was conducted to determine which HM samples were representative for analysis. Here a relatively complete investigation is presented following the collection of all currently available HM samples from large-scale cultivation areas covering major HM markets in China, with a few samples from Southeast Asia, without regard to possible pollution sources. For each available HM category in each HM market, at least one HM sample from each cultivation area was collected. A total of $1771 \mathrm{HM}$ samples from 503 major cultivation areas (Table S1.1, S1.2, appendix) were sampled for pesticide analysis, using either Liquid chromatography-tandem mass spectrometry (LC/MS-MS) (Shah et al., 2015) or Gas chromatography-tandem mass spectrometry (GC/MS-MS) (Cherta et al., 2013). A total of 136 pesticides (Table S2.1, appendix) with high toxicity and high detection rates were selected for

(1)


130

131

132

133

134

135

136

137

138

139

140

141

142

143

144

145

\subsection{Sample preparation and instrumentation}

The sample preparation protocol applied was slightly modified from a previously published protocol by

Waters Corporation (Waters Corporation, 2018). One gram of HM sample powder was weighed in a 50 $\mathrm{mL}$ centrifuge tube and then homogenized with $10 \mathrm{~mL}$ of water. The tube was shaken for 30 seconds using a hand-motion shaker (IKA VORTEX GENIUS 3, VG 3 S25). Subsequently, $10 \mathrm{~mL}$ of $1 \%$ glacial acetic acid in acetonitrile was added as an extraction solvent, followed by the addition of QuEChERS AOAC3 material (Waters DisQuE QuEChERS pouches, p/n 186006812). The tube was capped, vortexed for $1 \mathrm{~min}$, and centrifuged (Eppendorf, $5920 \mathrm{R}$ ) at $4500 \mathrm{RPM}$ for 5 minutes. One $\mathrm{mL}$ aliquot of the supernatant was removed and dried. The dried extract was then re-dissolved in $2 \mathrm{~mL}$ QuEChERS d-SPE tubule containing $50 \mathrm{mg}$ Primary Secondary Amine (PSA), $150 \mathrm{mg}$ anhydrous $\mathrm{MgSO}_{4}$, and $50 \mathrm{mg} \mathrm{C} 18$ followed by 30 seconds whirlpool and centrifugation (CK1060, Biotake Corporation) at 12000 RPM for 3 minutes. In order to detect different kinds of pesticides, LC-MS/MS or GC-MS/MS was applied separately for different HM samples. Therefore $0.1 \mathrm{~mL}$ supernatant was diluted with $0.9 \mathrm{~mL}$ of water and filtered with a microporous membrane $(0.22 \mu \mathrm{m})$ before LC-MS/MS analysis. While for GC-MS/MS analysis, $0.1 \mathrm{~mL}$ supernatant was transferred into a vial and diluted with $0.9 \mathrm{~mL}$ isooctane.

UPLC-MS/MS analysis was performed adopting Ultra Performance LC (UPLC) coupled to tandem quadrupole mass spectrometry (Xevo TQ-XS) with ACQUITY UPLC I-Class system. The chromatographic column (ACQUITY UPLC BEH C18 $2.1 \times 100 \mathrm{~mm}, 1.7 \mu \mathrm{m}$ ) was utilized for LC separation at $45{ }^{\circ} \mathrm{C}$. The injector was operated with $5 \mu \mathrm{L}$ volume, the flow rate was at $0.45 \mathrm{~mL} \cdot \mathrm{min}^{-1}$. 
151 The mobile phase consisted of $10 \mathrm{mM}$ ammonium acetate $(\mathrm{pH}=5)$ in water $(\mathrm{A})$ and $10 \mathrm{mM}$ ammonium

152 acetate $(\mathrm{pH}=5)$ in methyl alcohol (B). The gradient elution program was as follows: 0-0.25 min, $\mathrm{B}, 2 \%$;

$153 \quad 0.25-12.25 \mathrm{~min}, \mathrm{~B}, 2 \%-99 \% ; 12.25-13.00 \mathrm{~min}, \mathrm{~B}, 99 \% ; 13.00-13.01 \mathrm{~min}, \mathrm{~B}, 99-2 \% ; 13.01-17.00 \mathrm{~min}$,

$154 \mathrm{~B}, 2 \%$. The capillary voltage of $\mathrm{MS}$ was $3 \mathrm{KV}$ with a source temperature of $150{ }^{\circ} \mathrm{C}$ and desolventation

155 temperature of $500{ }^{\circ} \mathrm{C}$. The desolventation was run at the speed of $1000 \mathrm{~L} \cdot \mathrm{h}^{-1}$ while the cone at 150

$\mathrm{L} \cdot \mathrm{h}^{-1} \cdot{ }^{21}$ (Table S2.2, S2.4, appendix)

157

APGC-MS/MS analysis was employed by ESI/APGC+TQ-S platform using the Agilent 7890B system

(Agilent 7890B, Palo Alto, CA, USA) coupled to a triple quadrupole (QqQ) mass spectrometer (Xevo

TQ-S, Waters Corporation, Manchester, UK), operating in APGC mode. A fused silica DB-5MS

capillary column $(30 \mathrm{~m} \times 0.25 \mathrm{~mm}$ i.d. $\times 0.25 \mu \mathrm{m}$ film thickness $)(\mathrm{J} \& \mathrm{~W}$ Scientific, Folson, CA, USA)

microliter of the sample was injected. The GC inlet was operated in pulsed splitless mode and worked at $280{ }^{\circ} \mathrm{C}$. The oven temperature was programmed as follows: initial temperature $70{ }^{\circ} \mathrm{C}$ held for 1 $\min , 15{ }^{\circ} \mathrm{C} \cdot \min ^{-1}$ to $150{ }^{\circ} \mathrm{C}$ and $10^{\circ} \mathrm{C} \cdot \min ^{-1}$ to $300{ }^{\circ} \mathrm{C}$ held for $3 \mathrm{~min}$. A pulsed splitless injection was automatic dwell time (values ranging from 4 to $52 \mathrm{~ms}$ ) was applied in order to obtain at least 12 points per peak. The interface temperature was set to $300{ }^{\circ} \mathrm{C}$ using $\mathrm{N}_{2}$ as auxiliary gas at $250 \mathrm{~L} \cdot \mathrm{h}^{-1}$, a makeup gas at $270 \mathrm{~mL} \cdot \mathrm{min}^{-1}$ and cone gas at $170 \mathrm{~L} \cdot \mathrm{h}^{-1}$. The APGC corona discharge pin was operated at $1.8 \mu \mathrm{A}$.

171 Water was used as a modifier when working under proton-transfer conditions was placed in an 
173 Targetlynx (a module of MassLynx) and Quanpedia database software (Cherta et al., 2013) (Table

174 S2.3, S2.5, appendix).

175

176

177

\subsection{Quantitative methods}

In our experiment, the mixture of Multi-residue Pesticide Standards for LC/MS-MS was bought from Waters® LC Multi-residue Pesticide Standards Kit, p/n 186007574 and for GC/MS-MS from Sigma Aldrich as reference substances. The $5 \mu \mathrm{g} \cdot \mathrm{mL}^{-1}$ multi-pesticide standards mixtures were prepared in acetonitrile and stored in a freezer at $-20{ }^{\circ} \mathrm{C}$. The working standard mixtures were prepared by volume dilution of stock solutions in hexane to prepare matrix-matched calibrants and in acetone for sample fortification (Cherta et al., 2013). The detailed standard curves, $\mathrm{R}^{2}$, retention time, quantification trace, and ion trace of each pesticide were listed in the appendix (Table S2.1-S2.5, appendix). The methodologies of LC/MS-MS and GC/MS-MS were modified from previously validated studies by Shah et al. (Shah et al., 2015) and Cherta et al. (Cherta et al., 2013). With respect to LC/MS-MS, the linearity range and standard calibration curves were established by spiking the pesticide mixtures from $0.00125 \mathrm{mg} \cdot \mathrm{kg}^{-1}$ to $0.32 \mathrm{mg} \cdot \mathrm{kg}^{-1}$ respectively. Quantification was performed against a matrix-matched calibration curve. All of the incurred pesticide residues detected were identified from comparison with the retention time and ion ratio from reference standards within the tolerance limit specified by the European Commission (EC, 2016). As for GC/MS-MS, the selected reaction monitoring (SRM) method was modified from the study by Cherta et al. to evaluate linearity, precision, and selectivity (Cherta et al., 2013). Linearity was tested by injecting standards in hexane at eight concentrations, 0.1 , $0.5,1,5,10,25,50$, and $100 \mathrm{ng} \cdot \mathrm{mL}^{-1}$, considered acceptable as regression coefficients were over 0.99 . Precision was estimated as repeatability in terms of relative standard deviation $(\mathrm{RSD}, \%)(n=6)$ 
195

196

197

198

199

200

201

202

203

204

205

206

207

208

209

calculated for each fortification level. The selectivity forevaluating each of the three specific SRM transitions measured was satisfactory. For the identification of pesticides, criteria were set for the ratio of the response obtained for the transitions measured (EC, 2011).

\subsection{Statistic analysis}

The lowest linearity range of this experiment is $0.02 \mathrm{mg} \cdot \mathrm{kg}^{-1}$. Only data equal to or above it were retained in data analysis (PAN, 2006). For detection rate and the over-limit ratio of 136 pesticides, the total number of samples detected with at least one pesticide, the number of samples detected of each pesticide, and the number of pesticides detected in single HM were calculated. In addition, to simplify comparisons HM was divided into five categories, according to the part of the plant used for medicinal properties: fructus \& semen, radix \& rhizoma, flos, folium \& cortex, and herba \& others. The numbers of pesticides in five medicinal properties of HM were aggregated by classes 1-5, 6-10, 11-15, 16-20, and $>20$, respectively, as well as detection frequency of six pesticide types.

The total number of samples detected with banned pesticides, the number of samples detected with each of the 35 pesticides, and the number of banned pesticides detected in a single sample were calculated.

To analyze the over-limit ratio of pesticide in HM, Maximum Residue Limits (MRLs) of pesticides is needed as a reference, yet the MRLs of 136 pesticides are not available in any existing regulation in any country. Therefore we calculated the MRLs of 136 pesticides in $182 \mathrm{HM}$ according to the 
216

217

218

219

220

221

222

223

224

225

226

227

228

229

230

231

232

233

234

European Pharmacopoeia (EP, 8th Edition) (Europe pharmacopoeia, 2014). If the pesticide limits are neither listed in EP nor Regulation (EC) No. 396/2005, its MRL shall be calculated using the following expression:

$\mathrm{MRL}=\frac{\mathrm{ADI} \times \mathrm{BW}}{\mathrm{IR}_{d} \times 100}$

Where ADI $\left(\mathrm{mg} \cdot \mathrm{kg}^{-1}\right)$ is the acceptable daily intake of each pesticide. BW is the body weight $60 \mathrm{~kg}$;

$\mathrm{IR}_{\mathrm{d}}$ refers to the daily dose of HM in kilograms and here we used maximal dose derived from CP 2015.

Besides, for pesticides whose ADIs are not available, default MRL $0.01 \mathrm{mg} \cdot \mathrm{kg}^{-1}$ was applied according to the regulation by EP. Additionally, banned pesticides identified in our experiment based on the stipulations of the EU, US, and China were defined as Not Detected (ND). (Table S3)

For pesticides whose ADIs are vacant but NOELs available, ADI was derived from NOEL by:

$\mathrm{ADI}=\frac{\mathrm{NOEL}}{\text { safety factor }}$

Where ADI is acceptable daily intake $\left(\mathrm{mg} \cdot \mathrm{kg}^{-1}\right)$; NOEL is no-observed-effect level; safety factor is set as 1000 (USFDA, 2018; Vania, 2000).

Times of highest concentrations detected over the MRLs were calculated by concentration of pesticides detected minus MRL calculated, then divided by MRL: 
237 Times over MRL $=\frac{\text { C-Limit }}{\text { Limit }}$

238

239

When calculating times of concentrations detected over the MRLs for ADI-vacant and banned pesticides, default MRL $0.01 \mathrm{mg} \cdot \mathrm{kg}^{-1}$ was adopted.

\subsection{Three approaches of risk assessments}

Dietary exposure assessment (Bhandari et al., 2019) was employed to classify acceptable and unacceptable risks of pesticides in HMs posed to health due to elevated background of dietary exposure and risk-ranking score (VRC, 2007) to categorize and prioritize pesticides and HMs by ranking. Additionally, for the first time, patient health impact was characterized to combine both cancerogenic and non-cancerogenic risks, and ultimately connected these risks of pesticides interpreted to disease probability in a lifetime (Fantke, 2012). Hazard Quotient (HQ) and Hazard Index (HI) were applied to quantify acute, chronic, and cumulative risks posed by pesticide residues in HM for dietary exposure assessment (Bhandari et al., 2019). The risk-ranking score (S) for pesticides and Risk Index (RI) for HM were obtained by multiplying three groups of individual scores which represent the toxicity of the pesticide, the intake of pesticides, and the level of detectable residues (VRC, 2007). Also, we quantified potential patient intake caused by pesticide residues in HMs and related cancer and non-cancer aggregated effect estimates based on methods developed for comparative impact assessment, and combined both into a set of comparative patient health impact scores (IS), expressed as disability-adjusted life years (DALYs) (Fantke, 2012).

Using this approach, the absence of dietary exposure of certain pesticides due to lack of Acceptable 
259

260

261

262

263

264

265

266

267

268

269

270

271

$272 \quad \mathrm{HQ}_{\mathrm{a}}=\frac{\mathrm{ESTI}}{A R f D}$

Daily Intake (ADI) and Acute Reference Dose (ARfD) was compensated for by applying risk-ranking score and patient health impact characterization, which also aggregates both cancerous and non-cancerous effects. Therefore, comprehensive and detailed data on pesticide content in HM were obtained to support a robust interpretation of their impacts on health. Revealed here is a more critical cause of the adverse effects of HM.

\subsubsection{Dietary Exposure Assessment}

The Hazard Quotient (HQ) and Hazard Index (HI) were applied here to quantify adversity raised by pesticide residues in HM. The acute, chronic and cumulative risks were evaluated according to the following equations (Bhandari et al., 2019; Xiao et al., 2019; Zuo et al., 2019):

$\mathrm{ESTI}=\frac{\mathrm{Ef} \times \mathrm{Ed} \times \mathrm{IR}_{\mathrm{h}} \times C_{h}}{\mathrm{AT} \times \mathrm{BW}}$

274 Where ESTI $\left(\mathrm{mg} \cdot \mathrm{kg}^{-1} \cdot \mathrm{bw}^{-1}\right)$ is the estimated short-term intake, Ef is exposure frequency, here the $95^{\text {th }}$

275 percentile of annual consumption on HM was adopted which was set 90 days per year according to a questionnaire on HM consumption of 20917 people (Wang et al., 2017); Ed is the exposed days over a lifetime which is set as 20 years; $\mathrm{IR}_{\mathrm{h}}(\mathrm{kg})$ here refers to $95^{\text {th }}$ percentile of $\mathrm{HM}$ taken on a single day per person, which is set to $0.5 \mathrm{~kg}, \mathrm{C}_{\mathrm{h}}\left(\mathrm{mg} \cdot \mathrm{kg}^{-1}\right)$ is the highest residue level of pesticides; AT is the average 
279

280

281

282

283

284

285

286

287

288

289

290

291

292

$293 \quad \mathrm{HI}=\sum_{i=1}^{\mathrm{n}} H Q_{i}$

$\mathrm{HQ}_{\mathrm{c}}=\frac{\mathrm{EDI}}{\mathrm{ADI}}$

lifetime $=365$ days $\times 70$ years; BW is the mean body weight, set as $60 \mathrm{~kg}$. The estimate of pesticide intake in HM was then compared to the Acute Reference Doses (ARfD) (mg.kg ${ }^{-1}$ ) (Zuo et al., 2019).

$\mathrm{EDI}=\frac{\mathrm{Ef} \times \mathrm{Ed} \times \in \mathrm{IR}_{\mathrm{m}} \times C_{m}}{\mathrm{AT} \times \mathrm{BW}}$

Where EDI $\left(\mathrm{mg} \cdot \mathrm{kg}^{-1} \cdot \mathrm{bw}^{-1}\right)$ is the estimated daily intake; $\mathrm{IR}_{\mathrm{m}}(\mathrm{kg})$ here refers to the mean dosage of each $\mathrm{HM}$ which is $0.2 \mathrm{~kg}$ and $\mathrm{C}_{\mathrm{m}}\left(\mathrm{mg} \cdot \mathrm{kg}^{-1}\right)$ is the mean residue level of each pesticide in the single herb (Kumari et al., 2019).

$\mathrm{HQ}_{\mathrm{a}} \mathrm{s}$ and $\mathrm{HQ}_{\mathrm{c}} \mathrm{s}$ of each pesticide in the same $\mathrm{HM}$ were summed up to give a cumulative hazard index of single $\mathrm{HM}$ as $\mathrm{HI}_{\mathrm{a}}$ and $\mathrm{HI}_{\mathrm{c}}$ (USEPA, 2002; EFSA, 2013):

294

295

An HQ of $<1$ or HI of $<1$ for any pesticide is often considered as acceptable; whereas an HQ or HI of $>1$ not. As the HQ or $\mathrm{HI}$ increases, the risk does as well. For $\mathrm{HI}_{\mathrm{a}}>1$ and $\mathrm{HI}_{\mathrm{c}}>1 \mathrm{HM}$, the contribution of each pesticide was calculated by $\left(\mathrm{HQ}_{\mathrm{a}} / \mathrm{HI}_{\mathrm{a}}\right) \times 100 \%$ and $\left(\mathrm{HQ}_{c} / \mathrm{HI}_{c}\right) \times 100 \%$ to explore which pesticide contributes the most to HM posing risks (USEPA, 2002; EFSA, 2013). 
299

300

301

302

303

304

305

306

307

308

309

310

311

312

313

314

315

316

317

318

319

\subsubsection{Risk-ranking score}

The risk-ranking score (S) is obtained by multiplying three groups of individual scores which represent the toxicity of the pesticide, the consumption of pesticides and the evidence of detected residues, calculated by the following equation (Nie et al., 2014; Li et al., 2018):

Overall Risk-ranking Score $(\mathrm{S})=(\mathrm{A}+\mathrm{B}) \times(\mathrm{C}+\mathrm{D}+\mathrm{E}) \times \mathrm{F}$

Where A refers to nature of the hazard, here refers to $\mathrm{LD}_{50}$ which determines the toxicity of pesticides

(Table S4, appendix); B is the potency of the substance, here refers to ADI of pesticides; C is exposure

1, here refers to the maximal dosage of HM (CP 2015); D is exposure 2, which is calculated by Frequency=times of pesticide application/planting days. Considering the data regarding the pesticide application in HM is not available, and in most cases pesticide is applied relatively less frequently in HM than in crops, the D value was considered less than $2.5 \%$ which signifies 0 for all cases. E is exposure 3, here refers to the chance of exposure of HMs to the population. Since HM could be applied in medicinal, dietary, and tea occasions, herbs with only one function are set with 1 point, with two functions 2 points and three functions 3 points; F refers to evidence for detected residues. (Tables S2.1, appendix)

For overall risk-ranking score of each herb, Risk Index (RI) is calculated by summing up $S$ of each pesticide in one single herb.

$\mathrm{RI}=\sum_{\mathrm{i}=1}^{n} S$ 
320

321

322

323

324

325

326

327

328

329

330

331

332

333

334

335

336

338

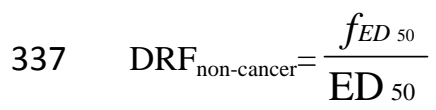

Where RI is the Risk Index of single HM, $S$ is the risk-ranking score of each pesticide, $n$ is the number of pesticides detected in one single herb. Sensitivity analyses were performed by estimating the contribution of $\mathrm{A}+\mathrm{B}, \mathrm{C}+\mathrm{D}+\mathrm{E}$ and $\mathrm{F}$ to the variance in the overall risk score by Spearman correlation coefficient analysis (Spss 17.0) (Li et al., 2018).

\subsubsection{Patient health impact assessment}

Patient health impact score (IS), expressed as disability-adjusted life year (DALY), was developed to evaluate health impact by multiplying IF, the human intake factor and EF, the human toxicological effect factor which reflects the change in lifetime disease probability due to change in lifetime intake of pesticides (Fantke et al., 2012; Frischknecht et al., 2016). DRF is the Human dose-response slope factor (incidence risk.kg a.i. intake ${ }^{-1}$ ), which can be derived for cancer effects from human or extrapolated from animal $\mathrm{TD}_{50}$ and for non-cancer effects from human $\mathrm{ED}_{50}$ or extrapolated from animal $\mathrm{ED}_{50}$, $\mathrm{NO}(\mathrm{A}) \mathrm{EL}, \mathrm{LO}(\mathrm{A}) \mathrm{EL}$ or $\mathrm{LD}_{50}$ by the following equations (Rosenbaum et al., 2011; Huijbregts et al., 2005). Only five $\mathrm{TD}_{50} \mathrm{~s}$ of carbaryl, methidathion, p,p'-DDD, p,p'-DDT, and piperonyl butoxide were available and have been certainly proven with cancerogenic effects. The lowest $\mathrm{ED}_{50}$ was applied to derive the highest Impact Score (IS) to be conservative.

$\mathrm{DRF}_{\text {cancer }}=\frac{f_{\mathrm{T} D} \mathrm{~T}_{50}}{\mathrm{TD}_{50}}$ 
339

$\mathrm{ED}_{50 \text { non-cancer }}=\frac{\mathrm{NOEL} \times f_{\text {NOEL }} \times \mathrm{BW} \times \mathrm{AT}}{f_{s} \times f_{\text {time }} \times f \frac{\mathrm{mg}}{\mathrm{kg}}}$

340

$\mathrm{ED}_{50 \text { non-cancer }}=\frac{\mathrm{LOAEL} \times f_{\text {LOAEL }} \times \mathrm{BW} \times \mathrm{AT}}{f_{s} \times f_{\text {time }} \times f \frac{m g}{k g}}$

341

342

$\mathrm{ED}_{50 \text { non-cancer }}=\frac{\mathrm{LD} 50}{26}$

$\mathrm{ED}_{50 \text { cancer }}=\frac{\mathrm{TD}_{50} \times \mathrm{BW} \times \mathrm{AT}}{f_{s} \times f \frac{m g}{k g}}$

$f_{E D 50}$ or $f_{T D 50}=0.5$ is the human response level corresponding to $\mathrm{ED}_{50}$ or $\mathrm{TD}_{50} ; \mathrm{ED}_{50}$ refers to the median

effective dose of pesticide which can induce $50 \%$ effects on exposed subjects; $\mathrm{TD}_{50}$ is a numerical

a lifetime, to induce tumors in half of the test animals; NOEL refers to no-observed-effect level;

$f_{\mathrm{NOEL}}=9$ is the NOEL-to- $\mathrm{ED}_{50}$ extrapolation factor (University of Hertfordshire, 2019); $f_{s}$ corrects for 
357

with $\mathrm{ED}_{50}$ and $\mathrm{EF}_{\text {cancer }}$ with $\mathrm{TD}_{50}$ were calculated. If more than one parameter exists per chemical

(NOEL or LOAEL or $\mathrm{LD}_{50}$ ), lowest converted human lifetime $\mathrm{ED}_{50}$ was applied;

From DRF and the estimated residue-related patient intake, we derived impact scores based on measured residues of pesticide active ingredients (a.i.) in different consumed herbs:

$\mathrm{IS}_{\mathrm{i}, \mathrm{h}, \mathrm{d}}=\mathrm{IR}_{\mathrm{i}, \mathrm{h}} \times \mathrm{C}_{\mathrm{i}} \times \mathrm{PF}_{\mathrm{i}, \mathrm{h}} \times \sum_{\mathrm{e}}\left(D R F_{i, e} \times S F_{e}\right) \times f \frac{k g}{m g} \times f \frac{\mu D A L Y}{D A L Y}$

$\mathrm{IS}_{\mathrm{i}, \mathrm{h}}=\mathrm{IR}_{\mathrm{i}, \mathrm{h}} \times \mathrm{Ef} \times \mathrm{Ed} \times \mathrm{C}_{\mathrm{i}} \times \mathrm{PF}_{\mathrm{i}, \mathrm{h}} \times \sum_{\mathrm{e}}\left(D R F_{i, e} \times S F_{e}\right) \times f \frac{k g}{m g} \times f \frac{\mu D A L Y}{D A L Y}$

$\mathrm{IS}_{\mathrm{h}}=\sum_{\mathrm{i}} I S_{i, h}=\mathrm{IR}_{\mathrm{i}, \mathrm{h}} \times \mathrm{Ef} \times \mathrm{Ed} \times \sum_{\mathrm{i}}\left(C_{i} \times P F_{i, h} \times \sum_{e}\left(D R F_{i, e} \times S F_{e}\right)\right) \times f \frac{k g}{m g} \times f \frac{\mu D A L Y}{D A L Y}$

Where $\mathrm{IS}_{\mathrm{i}, \mathrm{h}, \mathrm{d}}\left(\mu \mathrm{DALY} \cdot\right.$ person $\left.^{-1} \cdot \mathrm{d}^{-1}\right)$ refers to IS per person per day for single herb-pesticide pair; $\mathrm{IS}_{\mathrm{i}, \mathrm{h}}$ $\left(\mu \mathrm{DALY} \cdot\right.$ person $\left.^{-1}\right)$ is IS per person for a single herb-pesticide pair over lifetime and $\mathrm{IS}_{\mathrm{h}}$ $\left(\mu \mathrm{DALY} \cdot\right.$ person $\left.^{-1}\right)$ is IS per person for a single herb over lifetime; $\operatorname{IR}_{\mathrm{i}, \mathrm{h}}\left(\mathrm{kg} \cdot \mathrm{person}^{-1} \cdot \mathrm{d}^{-1}\right)$ is the individual daily intake dose of single herb, here we adopted the maximal dose from CP 2015 to be conservative; $\mathrm{C}\left(\mathrm{mg} \cdot \mathrm{kg}^{-1}\right)$ refers to the measured residual concentration of pesticide active ingredient $i$ in HM, we adopted maximal and mean concentration of each pesticide detected in single HM; Ef refers to the number of days of individual daily intake of each herb every year, here we assume an individual takes HM 90 days each year; Ed is the exposed days over lifetime, set as 20 years here (Wang et al., 2017); $\mathrm{PF}_{\mathrm{i}, \mathrm{h}}$ is food processing factor $\left(\mathrm{mg}_{\text {a.i. in processed herb }} \cdot \mathrm{kg}^{-1}\right.$ processed herb per $\mathrm{mg}_{\text {a.i. in harvested herb }} \cdot \mathrm{kg}^{-1}$ harvested herb), considering no official data is available, we assume $\mathrm{PF}=1 ; \mathrm{SF}_{\mathrm{e}}$ refers to severity factor $\left(\right.$ DALY·incidence $\left.{ }^{-1}\right)$ derived from general statistics about disease severity for cancer and non-cancer effects with 11.5 DALY.incidence ${ }^{-1}$ for cancer and 2.7 DALY·incidence ${ }^{-1}$ for non-cancer (Huijbregts et al., 2005); $f$ is the conversion factors adjusting for $\mathrm{kg}$ per $\mathrm{mg}\left(10^{-6}\right)$ and for $\mu$ DALY per DALY $\left(10^{6}\right)$, 
379

380

381

382

383

384

385

386

387

where $1 \mu \mathrm{DALY}=31.56$ seconds or 0.526 minutes of lost healthy life per person over lifetime. Both

$\mathrm{DRF}_{\text {non-cancer }}$ and $\mathrm{DRF}_{\text {cancer }}$ were derived and summed per pesticide to obtain $\mathrm{IS}_{\mathrm{i}, \mathrm{h}}$ per HM-pesticide and $\mathrm{IS}_{\mathrm{h}}$ per HM, both of which were calculated in three categories with maximal, mean and minimal concentrations of each pesticide detected.

\subsection{Data sources}

The indicators of pesticides including CAS number, pesticide type, substance group, $f_{\mathrm{s}}, f_{\text {time }}$, $\mathrm{TD}_{50}$ $\left(\mathrm{mg} \cdot \mathrm{kg}^{-1} \cdot \mathrm{day}^{-1}\right)$, ADI $\left(\mathrm{mg} \cdot \mathrm{kg}^{-1}\right)$, ARfD $\left(\mathrm{mg} \cdot \mathrm{kg}^{-1}\right)$, NOEL $\left(\mathrm{mg} \cdot \mathrm{kg}^{-1} \cdot \mathrm{d}^{-1}\right)$, NOAEL $\left(\mathrm{mg} \cdot \mathrm{kg}^{-1} \cdot \mathrm{bw}^{-1} \cdot \mathrm{d}^{-1}\right)$ LOAEL $\left(\mathrm{mg} \cdot \mathrm{kg}^{-1} \cdot \mathrm{d}^{-1}\right)$ and $\mathrm{LD}_{50}\left(\mathrm{mg} \cdot \mathrm{kg}^{-1}\right)$ were obtained from pesticide databases including Pesticide Properties Database (PPDB) (University of Hertfordshire, 2019), EU Pesticide Database (EC, 2016), Pesticide Action Network Pesticide Database (PAN) (PAN, 2018), Chemical book (Beijing Xilinbuke Network Technology Co., Ltd, 2016), Cancer Potency Database (CPDB) (The Carcinogenic Potency Project, 2007), Toxicology Data Network from U.S. National Library of Medicine (TOXNET IRIS) (TOXNET, 2019), and Foodmate (Foodmate, 2012). For indicators not available, national documents including The WHO Recommended Classification of Pesticides by Hazard And Guidelines to Classification 2009 (WHO, 2009), Acceptable Daily Intakes (ADI) for Agricultural and Veterinary Chemicals Used in Food Producing Crops or Animals (Australia) (Australia Pesticides and Veterinary Medicines Authority, 2017), National Food Safety Standard-Maximum Residue Limits for Pesticides in Food (China) (CFDA, 2016), Pesticide MRLs in Agricultural Commodities (South Korea) were referred to (Ministry of Food and Drug Safety, 2019). If data neither available in the databases nor the documents, articles containing relative parameters were applied. All data were collected based on 
400

401

402 The classification of pesticides by hazard was categorized according to WHO Class which defines

403

404

405

406

407

408

409

410

411

412

413

414

415

416

417

(The R Core Team, 2019).

(moderately hazardous, $50<\mathrm{LD}_{50}<2000$ ) and III (slightly hazardous with $\mathrm{LD}_{50}$ Over 2000) (WHO,

2009). For exposure time, one-time admission or 1 week is considered acute, 2-6 weeks exposure is

subacute, 7-28 weeks exposure is subchronic and 1-2 years exposure is considered chronic (Vermeire,

2001; Fantke, 2011). HM was classified into five categories according to which part of herbal plants

were applied for medicinal use. For information regarding five medicinal properties and dosage of HM,

Chinese Pharmacopoeia (CP, 2015 edition) was referred to. For information on HM not available in CP,

the Encyclopedia of Chinese Herbal Medicine was considered (Nanjing University of Chinese

Medicine, 2006). For NOEL only available with unit ppm diet, according to Food additives Guidelines

for the preparation of toxicological working papers for the Joint FAO/WHO Expert Committee on Food

Additives (2000), it is converted to $\mathrm{mg} \cdot \mathrm{kg}^{-1} \cdot \mathrm{bw}^{-1}$ per day (rat 0.05 , rabbit 0.025 , and dog 0.03 )

(FAO/WHO, 2000).

Figures were plotted using OriginPro 2018C (64-bit) SR1b 9.5.1.195 (One Roundhouse Plaza

OriginLab Corporation Northampton, MA 01060 USA) and $R$ language [no IDE (integrated development environment), $R$ from the linux terminal $\mathrm{R}$ version 3.5.1. (2018-007-02)-- "Feather Spray" Copyright (C) 2018, the $R$ Function for Statistic Computing Platform: x86_64-pc-linux-gnu (64-bit)] 
422

423

424

425

426

427

428

429

430

431

432

433

434

435

436

437

438

439

440

441

\section{Results}

\subsection{Detection of 136 pesticides in $1771 \mathrm{HM}$ samples}

Totally 98 pesticides in $1000 \mathrm{HM}$ samples were detected by LC/MS-MS and 44 in 771 samples by GC/MS-MS, therefore a total of 136 pesticides (6 in common) were determined in 1771 samples with concentrations ranging from $0.02 \mathrm{mg} \cdot \mathrm{kg}^{-1}$ to $20.00 \mathrm{mg} \cdot \mathrm{kg}^{-1}$ (linearity range). In total, 136 pesticides, including 35 banned, five extremely toxic, and 20 highly toxic, were detected in $1771 \mathrm{HM}$ samples by either LC/MS-MS or GC/MS-MS (Table S2.1, S2.2, appendix).

\subsubsection{Detection rate of pesticides in herbal medicines}

Totally $88.03 \%$ (1559) samples were detected with at least one pesticide in 1771 samples (Fig.1). The detection rate of each pesticide ranges from $0.10 \%$ to $36.70 \%$ in the total of 98 pesticides detected by LC-MS and from $0.13 \%$ to $44.62 \%$ in 44 pesticides by GC-MS. Bifenthrin was detected in $44.62 \%$ samples, followed by diphenylamine (42.41\%), and metolachlor (41.50\%). Additionally, $51.38 \%$ of the total samples were detected with three or more pesticides, $24.45 \%$ with five or more pesticides, and 69.79\% with one to five different pesticides. Notably, five HMs were detected with over 21 different kinds of pesticides. Chrysanthemi flos was detected with 37 different kinds of pesticides, followed by Crataegi fructus (29), and Alpiniae oxyphyllae fructus (27). $48.56 \%$ of the pesticide residue contamination was located in the radix \& rhizoma of HMs, followed by fructus \& semen (29.83\%), and folium \& cortex $(8.98 \%)$. Insecticides $(45.42 \%)$ and fungicides $(33.69 \%)$ are most frequently detected pesticides (Table S 5,6,7, appendix). 
442

443

444

445

446

447

448

449

450

451
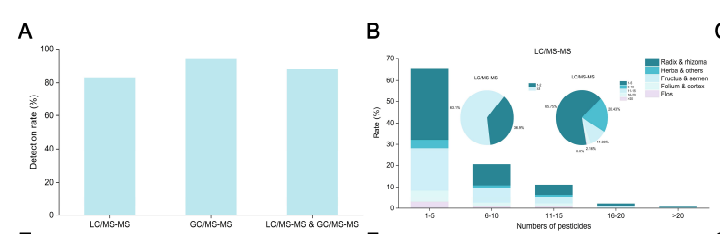

E
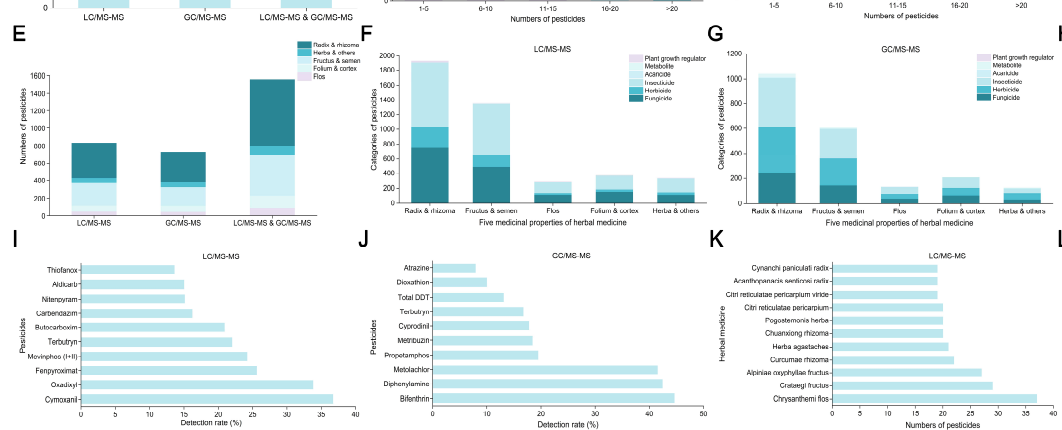

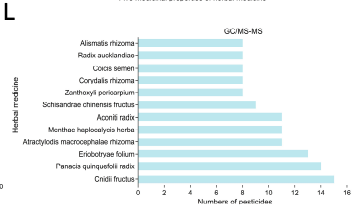

D
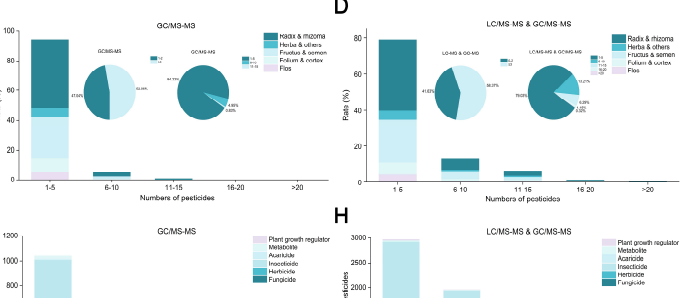

$\mathrm{H}$
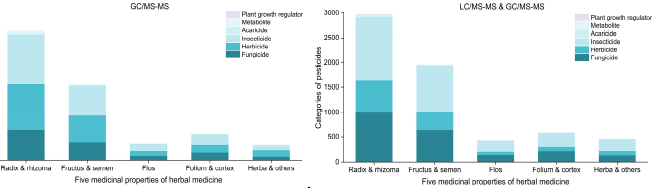

Fig.1 Detection rate of 136 pesticides in 1771 samples by LC/MS-MS and GC/MS-MS

(A)Total detection rate of overall pesticides by LC/MS-MS, GC/MS-MS and LC/MS-MS \& GC/MS-MS. (B) Detection rate of

five medicinal properties in different number of pesticides by LC/MS-MS. (C) Detection rate of five medicinal properties in different number of pesticides by GC/MS-MS. (D) Detection rate of five medicinal properties in different number of pesticides by LC/MS-MS \& GC/MS-MS. (E) Detection rate of five medicinal properties by LC/MS-MS, GC/MS-MS and LC/MS-MS \& GC/MS-MS. (F) Detection rate of six categories of pesticides in five medicinal properties by LC/MS-MS. (G) Detection rate of six categories of pesticides in five medicinal properties by GC/MS-MS. (H) Detection rate of six categories of pesticides in five medicinal properties by LC/MS-MS \& GC/MS-MS. (I) Detection rate of top ten pesticides by LC/MS-MS. (J) Detection rate of top ten pesticides by GC/MS-MS. (K) Number of pesticides detected in top ten HMs by LC/MS-MS. (L) Number of pesticides detected in top ten HMs by GC/MS-MS.

\subsubsection{Detection rate of banned pesticides}

The detection rate of banned pesticides in 1000 samples by LC/MS-MS was $57.70 \%$ and $23.87 \%$ in 771 samples by GC/MS-MS, so the total detection rate of banned pesticides was $42.97 \% \%$ in 1771 samples (Fig.2). The detection rate of 21 banned pesticides by LC/MS-MS ranged from $0.10 \%$ to $24.20 \%$, and 15 banned pesticides by GC/MS-MS ranged from $0.13 \%$ to $13.10 \%$. Mevinphos (I+II) was the banned pesticide with the highest detection rate (24.20\%), followed by carbendazim (16.20\%), 
459

460

461

462

463

464

465

466

467

aldicarb (15.00\%), and total DDT (13.10\%). 149 samples were detected with 3 or more banned pesticides. $47.44 \%$ of the samples detected with banned pesticides were found in radix \& rhizoma and $30.62 \%$ in fructus \& semen, $8.67 \%$ in folium \& cortex, $7.88 \%$ in herba \& others, and $5.39 \%$ in flos.

Crataegi Fructus were detected with ten different kinds of banned pesticides, followed by Herba agastaches (9), and Dendrobii caulis (8) (Table S5, S6, appendix).

Fig.2 Detection rate of banned pesticide by LC/MS-MS, GC/MS-MS and LC/MS-MS \& GC/MS-MS

(A) Total detection rate of banned pesticides by LC/MS-MS, GC/MS-MS and LC/MS-MS \& GC/MS-MS. (B) Detection rate of top ten banned pesticides by LC/MS-MS. (C) Detection rate of top ten banned pesticides by GC/MS-MS. (D) HMs with over seven banned pesticides detected by LC/MS-MS. (E) HMs with over three banned pesticide detected by GC/MS-MS. (F) HMs with over six banned pesticide detected by LC/MS-MS \& GC/MS-MS (F) Proportion of five medicinal properties detected with banned pesticides by LC/MS-MS. (H) Proportion of five medicine properties detected with banned pesticides by GC/MS-MS. (I) Proportion of five medicine properties detected with banned pesticides by LC/MS-MS \& GC/MS-MS.

\subsubsection{Over-limit ratio of pesticides in herbal medicines}


474 Based on the equation from the EP ( $8^{\text {th }}$ Edition $)$, all MRLs were set up for 136 pesticides in 182 HMs

475 (Table S3, appendix). Among which, 35 banned pesticides in EU, US, and China were set Not Detected

476 (ND), eight pesticides in lack of ADI were set as $0.01 \mathrm{mg} \cdot \mathrm{kg}^{-1}$, and the MRLs ranged from 0.0005

$477 \mathrm{mg} \cdot \mathrm{kg}^{-1}$ (Dicrotophos) to $3120.00 \mathrm{mg} \cdot \mathrm{kg}^{-1}$ (Chlorantraniliprole). Except for NDs and default MRLs

$478\left(0.01 \mathrm{mg} \cdot \mathrm{kg}^{-1}\right), 14 \mathrm{MRLs}(0.08 \%)$ were below $0.001 \mathrm{mg} \cdot \mathrm{kg}^{-1}, 181$ were between $0.01 \mathrm{mg} \cdot \mathrm{kg}^{-1}$ and

$4790.001 \mathrm{mg} \cdot \mathrm{kg}^{-1}, 1083$ between $0.01 \mathrm{mg} \cdot \mathrm{kg}^{-1}$ and $0.10 \mathrm{mg} \cdot \mathrm{kg}^{-1}, 6047$ between $0.10 \mathrm{mg} \cdot \mathrm{kg}^{-1}$ and 1.00

$480 \mathrm{mg} \cdot \mathrm{kg}^{-1}, 8399$ between $1.00 \mathrm{mg} \cdot \mathrm{kg}^{-1}$ and $10.00 \mathrm{mg} \cdot \mathrm{kg}^{-1}, 1467$ between $10.00 \mathrm{mg} \cdot \mathrm{kg}^{-1}$ and 100.00

$481 \mathrm{mg} \cdot \mathrm{kg}^{-1}, 96$ between $100.00 \mathrm{mg} \cdot \mathrm{kg}^{-1}$ and $1000.00 \mathrm{mg} \cdot \mathrm{kg}^{-1}$, and $3 \mathrm{MRLs}$ were above $1000.00 \mathrm{mg} \cdot \mathrm{kg}^{-1}$.

482 Comparing the concentrations of each pesticide detected in individual herb with MRLs calculated

483 according to EP, 1045 out of 1771 (59.01\%) samples were detected with concentration above the

484 threshold and the total over-limit ratio has reached $59.70 \%$ by LC/MS-MS and $58.11 \%$ by GC/MS-MS

485 (Fig.3). 
493

494

495

496

497

498

499

500

501

502

503

504

505

506

507

508

509

510

The concentrations of 49 pesticides have exceeded the EP standard by LC/MS-MS with over-limit ratios from $0.10 \%$ to $24.20 \%$ and 36 by GC/MS-MS from $0.13 \%$ to $19.33 \%$. The highest over-limit ratio was $24.20 \%$ by mevinphos (I+II), followed by propetamphos $(19.33 \%)$, carbendazim $(16.20 \%)$, aldicarb (15.00\%), and total DDT (13.10\%). Fifteen over-limit pesticides were detected in Crataegi fructus and ten in Pogostemonis herba. Totally 170 samples were detected with four to 15 pesticides exceeding the EP limit. Comparing detected residues with MRLs calculated according to EP, eight banned pesticides were detected with residues more than 500 times above the EP limit. Isocarbofos was 1997.0 times exceeding the EP limit, followed by carbendazim (1731.0), and total DDT (1521.0). (Table S5, 6, appendix).

\subsection{Risk assessments of pesticide contents in herbal medicines}

\subsubsection{Dietary Exposure Assessment}

The Hazard Quotient (HQ) and Hazard Index (HI) were applied here to quantify adversity raised by pesticide residues in herbal medicines Since 76 out of 136 pesticides are in lack of ARfD values, so only 60 pesticides participate in the acute risk assessment (Table S2.1, appendix). $72.95 \%$ of $\mathrm{HQ}_{\mathrm{a}}$ was below $0.01,22.98 \%$ ranged from 0.01 to $0.1,3.64 \%$ from 0.1 to 1.0 and only $0.43 \% \mathrm{HQ}_{\mathrm{a}}$ was above one (Fig.4). Pesticides carbofuran showed unacceptable risk in four HMs including Crataegi fructus $\left(\mathrm{HQ}_{\mathrm{a}}=10.80\right)$, Zanthoxyli pericarpium $\left(\mathrm{HQ}_{\mathrm{a}}=10.49\right)$, Lycii fructus $\left(\mathrm{HQ}_{\mathrm{a}}=1.61\right)$, and Corydalis bungeanae herba $\left(\mathrm{HQ}_{\mathrm{a}}=1.15\right)$, as well as pesticide Mevinphos $\mathrm{I}$ in Paridis rhizoma $\left(\mathrm{HQ}_{\mathrm{a}}=1.08\right)$ and Myristicae semen $\left(\mathrm{HQ}_{\mathrm{a}}=1.03\right)$, Methomyl in Rhei radix et rhizome $\left(\mathrm{HQ}_{\mathrm{a}}=1.30\right)$. The $\mathrm{HQ}_{\mathrm{a}} \mathrm{s}$ of other pesticides detected are all below one, thus considered as acceptable. Eight pesticides didn't take part in the chronic risk assessment for their ADIs were not available. $75.20 \% \mathrm{HQ}_{\mathrm{c}}$ was below $0.01,18.20 \%$ ranged from 0.01 to $0.1,6.41 \%$ ranged from 0.1 to 1.0 , and $0.20 \%$ was above one thus considered as 
515

516

517

518

519

520

521

522

523

524

525

526

527

528

529

530

531

532

unacceptable risk. The $\mathrm{HQ}_{\mathrm{c}} \mathrm{s}$ of carbofuran in Crataegi fructus and Zanthoxyli pericarpium were 4.32 and 1.52, respectively, of mevinphos I in Paridis rhizome, Myristicae semen, and Cnidii fructus were $2.83,2.72$, and 2.26 , respectively.

We calculated both acute cumulative risk and chronic cumulative risk for $182 \mathrm{HMs}$ with 60 pesticides for acute risk and 128 for chronic risk with data available. $4.40 \% \mathrm{HI}_{\mathrm{a}} \mathrm{s}$ was below $0.01 ; 61.54 \%$ ranged from 0.01 to $0.1 ; 28.57 \%$ is from 0.1 to 1.0 , and $5.49 \%$ was above one which considered as unacceptable. Totally ten $\mathrm{HMs}$ were presented with above-one $\mathrm{HI}_{\mathrm{a}} \mathrm{s}$, and Crataegi fructus ranked the top $\left(\mathrm{HI}_{\mathrm{a}}=12.09\right)$, followed by Zanthoxyli pericarpium $\left(\mathrm{HI}_{\mathrm{a}}=11.54\right)$, Lycii fructus $\left(\mathrm{HI}_{\mathrm{a}}=1.86\right)$, Corydalis bungeanae herba $\left(\mathrm{HI}_{\mathrm{a}}=1.48\right)$, Chrysanthemi flos $\left(\mathrm{HI}_{\mathrm{a}}=1.43\right)$, Myristicae semen $\left(\mathrm{HI}_{\mathrm{a}}=1.42\right)$, Rhei Radix et Rhizoma $\left(\mathrm{HI}_{\mathrm{a}}=1.41\right)$, Ganoderma $\left(\mathrm{HI}_{\mathrm{a}}=1.15\right)$, Paridis rhizome $\left(\mathrm{HI}_{\mathrm{a}}=1.14\right)$, and Cnidii fructus $\left(\mathrm{HI}_{\mathrm{a}}=1.07\right)$. While for chronic cumulative risk assessment, $1.65 \% \mathrm{HI}_{\mathrm{c}} \mathrm{s}$ was below $0.01,17.03 \%$ ranged from 0.01 to $0.1,68.68 \%$ ranged from 0.1 to 1.0 , and $12.64 \%$ was above one and considered unacceptable. The $\mathrm{HI}_{\mathrm{c}} \mathrm{s}$ of a total of $23 \mathrm{HMs}$ were above one, with Crataegi fructus $\left(\mathrm{HI}_{\mathrm{c}}=6.62\right)$ ranking the top, followed by Myristicae semen $\left(\mathrm{HI}_{\mathrm{c}}=3.51\right)$, and Zanthoxyli pericarpium $\left(\mathrm{HI}_{\mathrm{c}}=3.38\right)$.

Mevinphos I contributed $72.98 \%$ in the total $\mathrm{HI}_{\mathrm{a}}$ of Cnidii fructus, $72.41 \%$ in Myristicae semen, and 94.41\% in Paridis rhizome, while carbofuran contributed $77.95 \%$ in Corydalis bungeanae herba, $89.35 \%$ in Crataegi fructus, $86.84 \%$ in Lycii fructus, and $90.89 \%$ in Zanthoxyli pericarpium. Carbendazim contributed $44.35 \%$ in Ganoderma, Chlorpyrifos contributed $47.69 \%$ in Chrysanthemi flos, and Methomyl contributed $92.14 \%$ in Rhei radix et rhizoma. As per $\mathrm{HI}_{\mathrm{c}} \mathrm{s}, \quad$ coumaphos, isocarbofos, mevinphos I, carbofuran, propetamphos, methidathion, and chlorpyrifos were the chief pesticides made 
the major contributions in $\mathrm{HI}_{\mathrm{c}}>1 \mathrm{HMs}$. Conclusively, $\mathrm{HMs}$ Crataegi fructus and Zanthoxyli

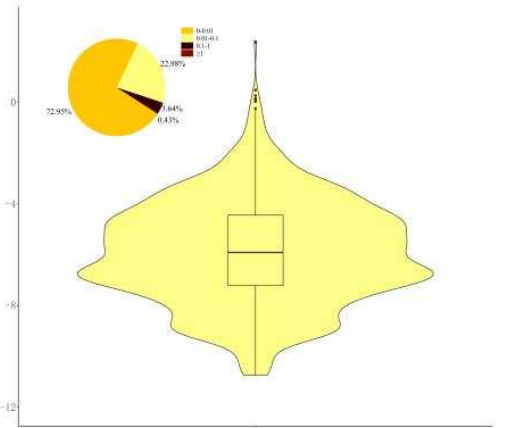

C

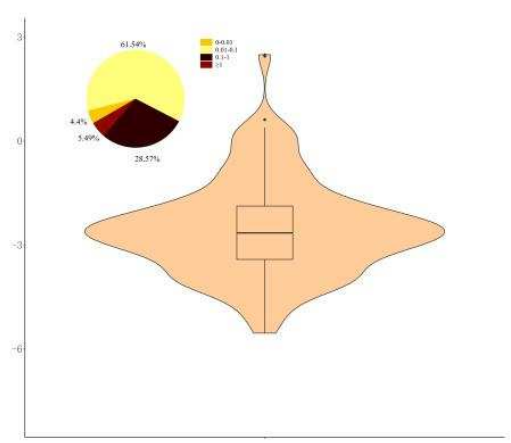

$E$

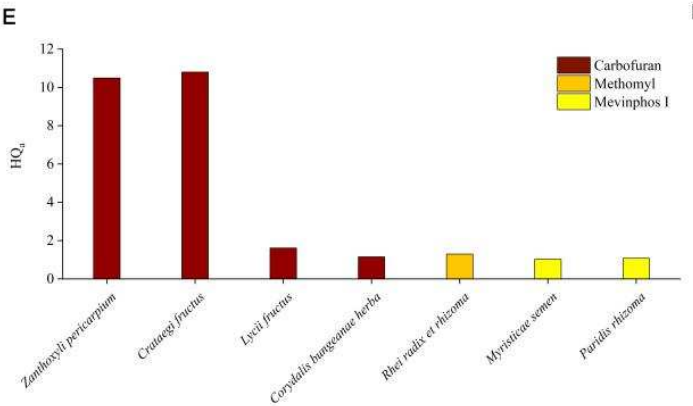

G

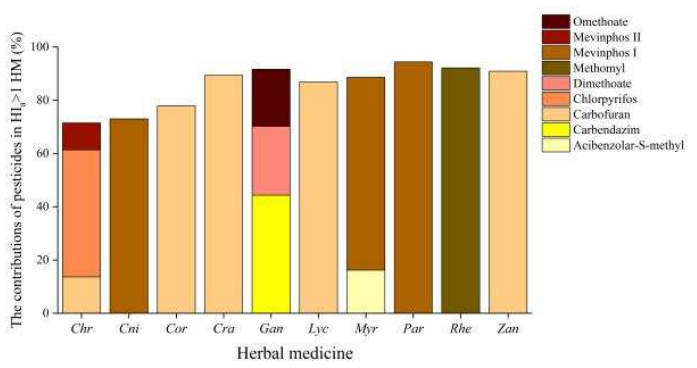

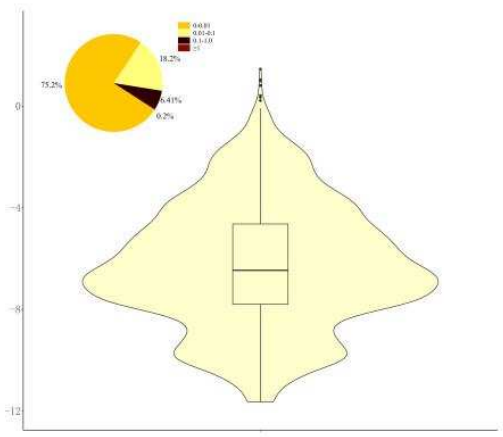

D

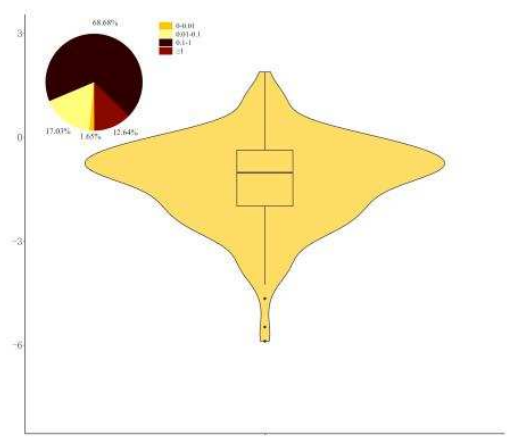

F

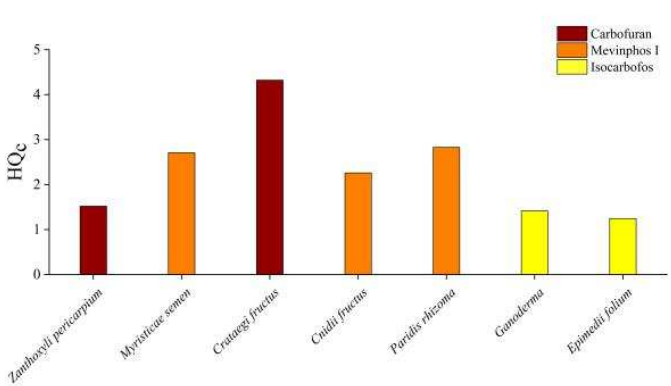

"

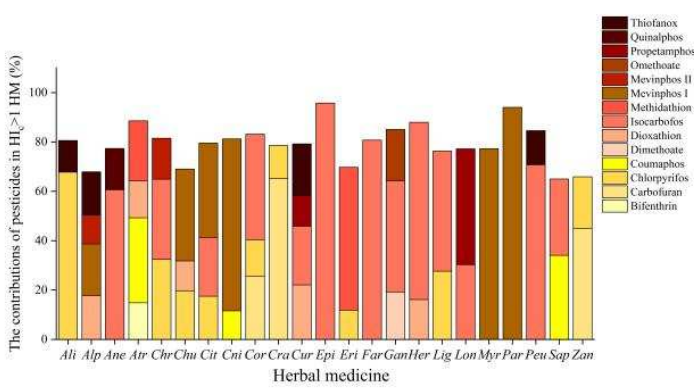


(A) Violin plot and proportion of acute hazard quotient $\left(\mathrm{HQ}_{\mathrm{a}}\right)$. (B) Violin plot and proportion of chronic hazard quotient $\left(\mathrm{HQ}_{\mathrm{c}}\right)$.

(C) Violin plot and proportion of acute hazard index $\left(\mathrm{HI}_{\mathrm{a}}\right)$. (D) Violin plot and proportion of chronic hazard index $\left(\mathrm{HI}_{\mathrm{c}}\right)$. (E)

oxyphyllae fructus, Ane for Anemarrhenae rhizoma, Atr for Atractylodis macrocephalae rhizoma, Chu for Chuanxiong rhizoma,

Cit for Citri reticulatae pericarpium, Cur for Curcumae longae rhizoma , Epi for Epimedii folium, Eri for Eriobotryae folium, Far

for Farfarae flos, Her for Herba sea radix amaranthi viridis, Lig for Ligustri lucidi fructus, Lon for Longan arillus, Peu for

\subsubsection{Risk-ranking Score}

The risk-ranking scores (S) of 136 pesticides detected in $182 \mathrm{HMs}$ all participated in the analysis, as 
564

565

566

567

568

569

570

571

572

573

574

575

576

577

578

579

580

581

582

583

584

585

Dendrobii caulis (1315) and Saposhnikoviae radix (1144) Additionally, the level of detection ( $\mathrm{p}=0.783$ )

contributed the most to the risk-ranking, followed by the toxicity of pesticide $(\mathrm{p}=0.691)$, and the least relevant was HM consumption ( $\mathrm{p}=0.370)$ according to Spearman correlation coefficients. For HMs with RI over 1000, we calculated the proportion of S of each pesticide contributed to its RI, the result is in accordance with the contributions of pesticides in $\mathrm{HMs}$ derived through $\mathrm{HQ}_{\mathrm{a}} / \mathrm{HI}_{\mathrm{a}}$ and $\mathrm{HQ}_{\mathrm{c}} / \mathrm{HI}_{\mathrm{c}}$. (Table 1, 2, Fig.S1, S3, S5 appendix).

\subsubsection{Patient health impact assessment}

Same as the risk-ranking score above, with three approaches of $\mathrm{ED}_{50}$ calculations, all pesticides detected were included in patient health impact assessment. With minimal concentrations detected of each pesticide in each HM, the $\mathrm{IS}_{\mathrm{i}, \mathrm{h,d}}$ ranged from 3.51E-07 to $1.52 \mu \mathrm{DALY} \cdot$ person $^{-1} \cdot \mathrm{d}^{-1}$; with mean concentrations, it ranged from $6.11 \mathrm{E}-07$ to $1.58 \mu \mathrm{DALY} \cdot \operatorname{person}^{-1} \cdot \mathrm{d}^{-1}$; with maximal concentrations, it ranged from $6.11 \mathrm{E}-07$ to $2.05 \mu \mathrm{DALY} \cdot$ person $^{-1} \cdot \mathrm{d}^{-1}$. The average value of all min $\mathrm{IS}_{\mathrm{i}, \mathrm{h,d}}$ was 0.01 $\mu$ DALY $\cdot \operatorname{person}^{-1} \cdot \mathrm{d}^{-1}$ and the median value was $8.02 \mathrm{E}-04 \mu \mathrm{DALY} \cdot$ person $^{-1} \cdot \mathrm{d}^{-1}$. For mean $\mathrm{IS}_{\mathrm{i}, \mathrm{h}, \mathrm{d}}$, the average and median were 0.01 and $0.001 \mu \mathrm{DALY} \cdot \operatorname{person}^{-1} \cdot \mathrm{d}^{-1}$, respectively, while for maximal $\mathrm{IS}_{\mathrm{i}, \mathrm{h,d}}$, 0.02 and $0.001 \mu \mathrm{DALY} \cdot$ person $^{-1} \cdot \mathrm{d}^{-1}$. As per $\mathrm{IS}_{\mathrm{i}, \mathrm{h}}$, the minimal values ranged from 0.0006 to 2729.59 $\mu \mathrm{DALY} \cdot$ person $^{-1}$, the mean from 0.001 to $2837.91 \mu \mathrm{DALY} \cdot$ person $^{-1}$, and the maximal from 0.001 to 3682.78 $\mu \mathrm{DALY} \cdot$ person $^{-1}$. The average values of minimal, mean, and maximal $\mathrm{IS}_{\mathrm{i}, \mathrm{h}}$ were 19.06, 23.00, and $28.19 \mu \mathrm{DALY} \cdot$ person $^{-1}$, respectively. Their median values were $1.44,2.22$, and 2.62 $\mu$ DALY person ${ }^{-1}$. The ranges of minimal, mean, and maximal $\mathrm{IS}_{\mathrm{h}}$ were from 1.93 to 3945.40 $\mu \mathrm{DALY} \cdot$ person $^{-1}$, from 1.97 to $3972.07 \mu \mathrm{DALY} \cdot$ person $^{-1}$, and from 2.00 to $4287.78 \mu \mathrm{DALY} \cdot$ person $^{-1}$. 
586

587

588

589

590

591

592

593

594

595

596

597

598

599

600

601

602

603

604

605

606

607

210.22, 269.63, and 326.58 $\mu \mathrm{DALY} \cdot$ person $^{-1}$. With min and mean $\mathrm{IS}_{\mathrm{h}}$, Coicis semen ranked the top $\left(\min I S_{h}=3945.40 \mu D A L Y \cdot\right.$ person $^{-1}$, mean $I_{\mathrm{h}}=3972.07 \mu$ DALY person $^{-1}$. While with max $I_{\mathrm{h}}$, Chuanmingshinis violacei presented with the highest $\mathrm{IS}_{\mathrm{h}}\left(4287.78 \mu \mathrm{DALY} \cdot\right.$ person $\left.^{-1}\right)$. Pesticide o,p'-DDT in Coicis semen also ranked the top in $\min \mathrm{Is}_{\mathrm{i}, \mathrm{h}}\left(2729.58 \mu \mathrm{DALY} \cdot\right.$ person $\left.^{-1}\right)$, yet in Radix chuanmingshinis violacei ranked the top in both mean and max $\mathrm{Is}_{\mathrm{i}, \mathrm{h}}$ (mean $\mathrm{Is}_{\mathrm{i}, \mathrm{h}}=2837.91$ $\mu \mathrm{DALY} \cdot$ person $^{-1}, \max _{\mathrm{IS}} \mathrm{S}_{\mathrm{i}, \mathrm{h}}=3682.78 \mu \mathrm{DALY} \cdot$ person $^{-1}$ ). $($ Table 1, 2, Fig.S2, S4, S5, appendix).

\section{Discussion}

In total, 136 pesticides including 35 banned, five extremely toxic, and 20 highly toxic were detected in 1771 HM samples by either LC/MS-MS or GC/MS-MS. Summarizing results from both LC/MS-MS and GC/MS-MS experiments, at least $88.03 \%$ (1559) samples were present with pesticide residues and $59.01 \%$ (1045) samples with over-limit pesticides levels. Furthermore, 35 banned pesticides were detected in at least $42.97 \%$ samples (761), of which mevinphos and total dichlorobiphenyl trichloroethane (DDT) accounted for 24.20\% (242 of 1000 samples by LC/MS-MS) and 13.10\% (101 of 771 samples by GC/MS-MS), respectively (Fig.1, 2, 3). Additionally, eight banned pesticides appeared at concentration levels over 500 times higher than the EP limit (Table 1). Chrysanthemi flos were detected with 37 pesticides (eight over-limit and seven banned), followed by Crataegi fructus (29 pesticides) and Alpiniae oxyphyllae fructus (27 pesticides) (Table 2). Our findings indicated pesticides were most widely detected in radix \& rhizoma $(48.62 \%, \mathrm{n}=1559)$ and the least in flos $(5.77 \%, \mathrm{n}=1559)$ (Table S7.1, appendix). Pesticides presenting the highest risk belonged to the organophosphorus insecticide group (Fig.5). Insecticides and fungicides were those most frequently detected (45.42\% and 33.69\%, respectively, $\mathrm{n}=6387$ ) (Table S7.2, appendix). 
609 As per risk assessments, pesticide carbofuran in Crataegi fructus, Lycii fructus, Zanthoxyli pericarpium,

610 and Corydalis bungeanae Herba, pesticide mevinphos in HM Myristicae semen, Cnidii fructus and

611 Paridis rhizoma, Methomyl in Rhei radix et rhizoma, and isocarbofos in Ganoderma and Epimedii

612 folium posed unacceptable acute or chronic risk $\left(\mathrm{HQ}_{\mathrm{a}}>1\right.$ or $\left.\mathrm{HQ}_{\mathrm{c}}>1\right)$. Additionally, $25 \mathrm{HM}\left(\mathrm{HI}_{\mathrm{a}}>1\right.$ or

$613 \mathrm{HI}_{\mathrm{c}}>1$ ) posed unacceptable cumulative risk according to dietary exposure assessment (Table 1, 2, Fig.4,

614 appendix). Carbofuran-3-hydroxy and leptophos appeared with the highest risk-ranking score ( $\mathrm{S}=140)$,

615 while Crataegi fructus occupied the top in Risk Index (RI=1925) (Table 1, 2, Fig.S1, S4, S5, appendix),

616 whereas pesticide o,p'-DDT and HM Radix chuanmingshinis violacei and Coicis semen posed the most

617 severe risk according to patient health impact approach (Table 1, 2, Fig.S2, S4, S5, appendix).

618 Mevinphos, carbofuran, DDTs, leptophos, and methomyl are all banned pesticides and highly toxic

619 (Fig.5). Crataegi fructus ranked high in three assessments and it belongs to the fructus \& semen

620 category (Table 2), thus considered the most risk-inducing HM in need of special surveillance and 621 monitoring.

622 Table 1: Summary of pesticides ranking the top in detection and risk assessments

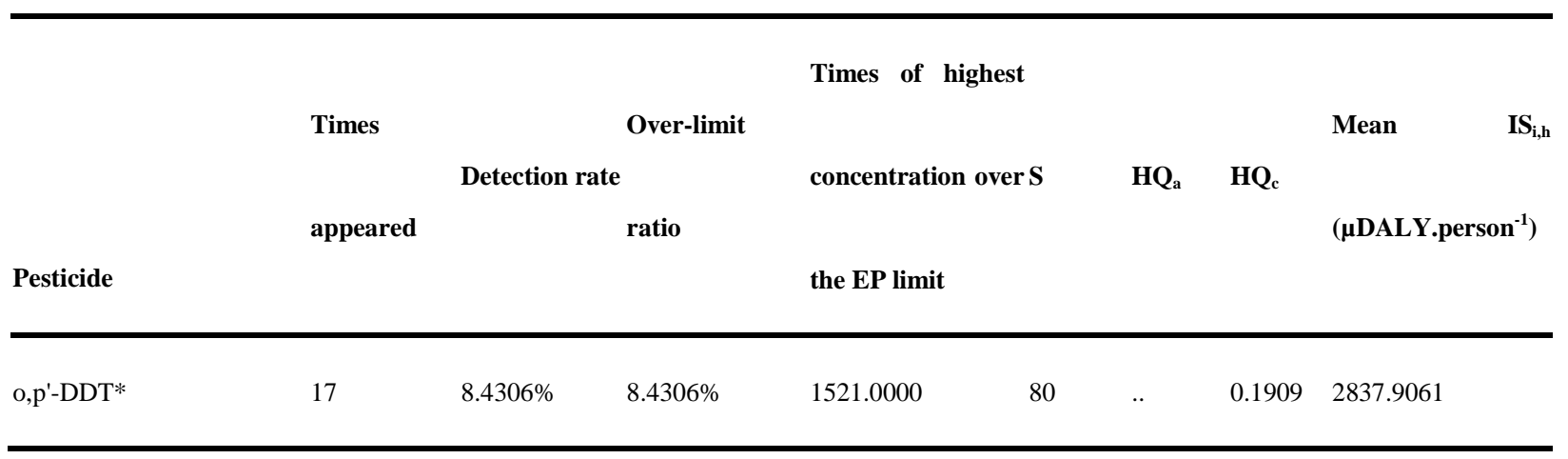




\begin{tabular}{|c|c|c|c|c|c|c|c|c|}
\hline \multicolumn{9}{|c|}{ loumal Pre-proof } \\
\hline Mevinphos I* & 10 & $21.6000 \%$ & $21.6000 \%$ & 549.0000 & 120 & 1.0763 & 2.8274 & 633.6051 \\
\hline $\mathrm{o}, \mathrm{p}^{\prime}-\mathrm{DDD}{ }^{*}$ & 10 & $5.8366 \%$ & $5.8366 \%$ & 943.0000 & 60 & .. & 0.2217 & 1155.3816 \\
\hline Carbofuran* & 6 & $2.4000 \%$ & $2.4000 \%$ & 275.0000 & 100 & 10.8023 & 4.3209 & 298.9317 \\
\hline Aldicarb* & 5 & $15.0000 \%$ & $15.0000 \%$ & 61.0000 & 120 & 0.1213 & 0.0438 & 1155.3816 \\
\hline Isocarbofos* & 5 & $10.4000 \%$ & $10.4000 \%$ & 1997.0000 & 100 & .. & 1.4153 & 444.0290 \\
\hline p,p'-DDT* & 4 & $0.1297 \%$ & $0.1297 \%$ & 409.0000 & 64 & .. & 0.0963 & 952.4230 \\
\hline Carbendazim* & 3 & $16.2000 \%$ & $16.2000 \%$ & 1731.0000 & 40 & 0.5084 & 0.2034 & 625.3503 \\
\hline Carbofuran-3-hydroxy* & 3 & $1.3000 \%$ & $1.3000 \%$ & 117.2000 & 140 & .. & .. & 61.5434 \\
\hline Bifenthrin & 2 & $44.6174 \%$ & $11.6732 \%$ & 18.1200 & 45 & 0.2806 & 0.1551 & 137.8599 \\
\hline Methomyl* & 2 & $0.4000 \%$ & $0.4000 \%$ & 550.8000 & 100 & 1.2958 & 0.5183 & 174.3136 \\
\hline Propetamphos & 2 & $19.4553 \%$ & $19.3256 \%$ & 78.9167 & 60 & .. & 0.6661 & 314.1194 \\
\hline Temephos* & 2 & $11.3000 \%$ & $11.3000 \%$ & 5.0000 & 40 & .. & 0.0001 & 1.4442 \\
\hline
\end{tabular}

\section{3}

624 Arranged in descending order by times appeared in both top detection and risk assessment lists. Pesticides marked with * are

625 banned pesticides (ND)... means no data obtained due to lack of ADI or ARfD.

626 We selected pesticides appeared over once in the top ten detection list by respectively summarizing the top ten detected, banned

627 and over-limit pesticides as well as top ten regarding the maximal times over the limit comparing to MRLs derived by EP

628 equation. We also collected those with $H Q_{a}>1$, and $H_{Q_{c}}>1$ pesticides in dietary exposure assessment, $S=140$ in risk-ranking score,

629 and those ranking top 10 in minimal, mean and maximal $\mathrm{IS}_{\mathrm{i}, \mathrm{h}}$ (only mean $\mathrm{IS}_{\mathrm{i}, \mathrm{h}}$ shown here) in patient health impact assessment.

630 Totally 13 pesticides were summarized in the table above. Of the 13 pesticides, 11 are banned, 11 are insecticides, one is

631 fungicide, and one is metabolite. Four of them belong to organophosphorus groups and another four are carbamates. Two of them

632 are extremely toxic and four are highly toxic. o,p'-DDT appeared 17 times in the top ranking pesticides which indicated that it is 
633

the pesticide posing the most widely and serious risk in herbal medicines, followed by Mevinphos I (10 times) and o,p'-DDD (10

634

times).

635

Table 2: Summary of herbal medicines ranking the top in detection and risk assessments

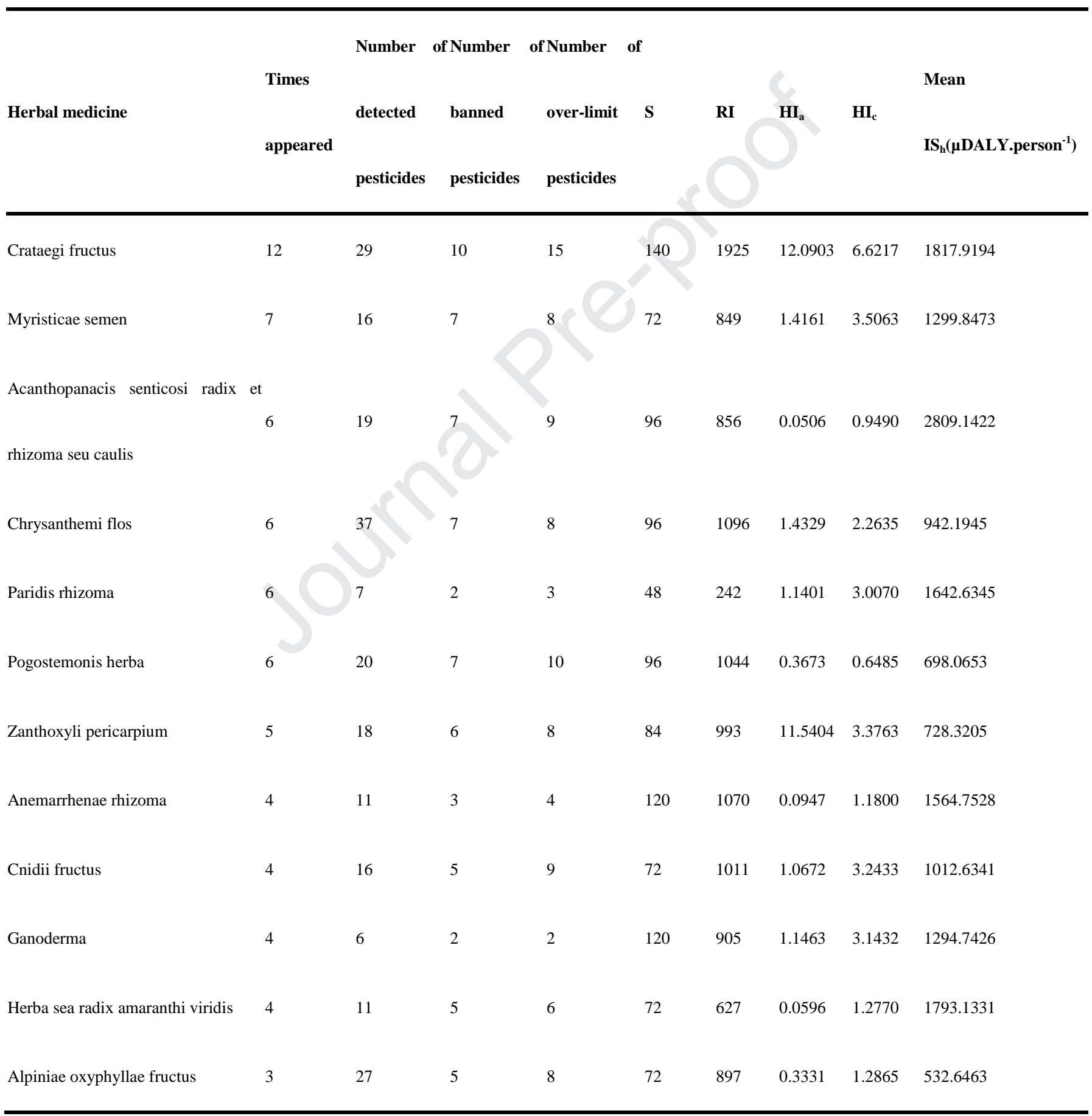




\begin{tabular}{|c|c|c|c|c|c|c|c|c|c|}
\hline Coicis semen & 3 & 8 & 2 & 5 & 120 & 910 & 0.0709 & 0.4822 & 3972.0719 \\
\hline Corydalis bungeanae herba & 3 & 12 & 4 & 6 & 84 & 621 & 1.4762 & 1.5270 & 932.4413 \\
\hline Cynanchi paniculati radix et rhizoma & 3 & 19 & 7 & 8 & 96 & 728 & 0.1060 & 0.7369 & 377.2584 \\
\hline Dendrobii caulis & 3 & 16 & 8 & 8 & 120 & 1315 & 0.0510 & 0.7085 & 429.6968 \\
\hline Herba agastaches & 3 & 21 & 9 & 9 & 96 & 776 & 0.2731 & 0.7643 & 260.3077 \\
\hline Lycii fructus & 3 & 9 & 3 & 4 & 140 & 825 & 1.8569 & 0.7606 & 414.0162 \\
\hline Pruni semen & 3 & 9 & 2 & 3 & 72 & 492 & 0.1632 & 0.5377 & 2046.1625 \\
\hline Radix aucklandiae & 3 & 8 & 1 & 5 & 32 & 254 & 0.8530 & 0.5679 & 2081.1195 \\
\hline Radix chuanmingshinis violacei & 3 & 7 & 3 & 4 & 96 & 624 & 0.0666 & 0.2505 & 3439.8703 \\
\hline Pinelliae rhizoma & 2 & 4 & 1 & 2 & 64 & 256 & 0.0828 & 0.1028 & 1465.5672 \\
\hline Chuanxiong rhizoma & 2 & 20 & 4 & 7 & 96 & 952 & 0.4741 & 1.4029 & 629.7785 \\
\hline Citri reticulatae pericarpium & 2 & 20 & 5 & 6 & 96 & 756 & 0.9027 & 1.3135 & 333.3653 \\
\hline Epimedii folium & 2 & 16 & 5 & 7 & 96 & 704 & 0.0458 & 1.3005 & 359.1246 \\
\hline Eriobotryae folium & 2 & 13 & 4 & 8 & 112 & 720 & 0.3838 & 1.0552 & 483.3975 \\
\hline Rhei radix et rhizoma & 2 & 13 & 4 & 6 & 120 & 1015 & 1.4064 & 0.8296 & 707.8251 \\
\hline Saposhnikoviae radix & 2 & 11 & 3 & 5 & 96 & 1144 & 0.2038 & 2.5268 & 899.4202 \\
\hline Taraxaci herba & 2 & 13 & 5 & 6 & 140 & 1035 & 0.0327 & 0.0681 & 255.5016 \\
\hline
\end{tabular}

636

637 Arranged in descending order by times appeared in both top detection and risk assessment lists.

638 We selected HM appeared over once in the top ten detection list by respectively summarizing the top ten HM with number of

639 detected, banned and over-limit pesticides, as well as $\mathrm{HI}_{\mathrm{a}}>1$ and $\mathrm{HI}_{\mathrm{c}}>1 \mathrm{HM}$ in dietary exposure assessment, top ten with highest 
patient health impact assessment. Those appeared over once were included, and totally 29 HM were summarized here.

belongs to flos. Crataegi fructus appeared 12 times in the indicators of three risk approaches, seemingly the most risky herbal medicine due to pesticide contamination, followed by Chrysanthemi flos, Pogostemonis herba, Acanthopanacis senticosi radix et

These detected pesticides are likely to exhibit fetotoxic, embryotoxic, as well as immunotoxic effects, potentially causing irreversible organ damage and reproductive issues (The Carcinogenic Potency interfere with the endocrinium, causing endocrine and metabolic disruption (Beyond Pesticides, 2018). They may also damage the nervous system, causing neurodegenerative and hepatorenal disorders (Sahoo et al., 2010) (Fig.5). Widely used as insecticides, DDTs are chemically stable, possessing a half-life of degradation ( $\mathrm{DT}_{50}$ ) as long as 30 years, capable of inducing carcinogenic effects (Vašíčková et al., 2019). Other frequently detected pesticides are likely to impart multisystem damage to the human body (Fig.5). Organophosphorus pesticides, such as azinphos-ethyl, chlorpyrifos, famphur,

659 Central Nervous System (CNS) symptoms including restlessness and depression of the respiratory or cardiovascular system (Han et al., 2017). Meanwhile, aldicarb, DDTs, and parathion methyl are qualified as Persistent Organic Pollutants (POPs), notable in their reputation for persistent toxicity to 
662 humans and animals, and ability to be transported around the globe through atmospheric vectors (Chen

663

664

665

666 et al., 2015). Heretofore unknown synergistic effects due to the combined effect of mixing the pesticides found in this study could result in multisystem dysfunctions yet to be discovered.

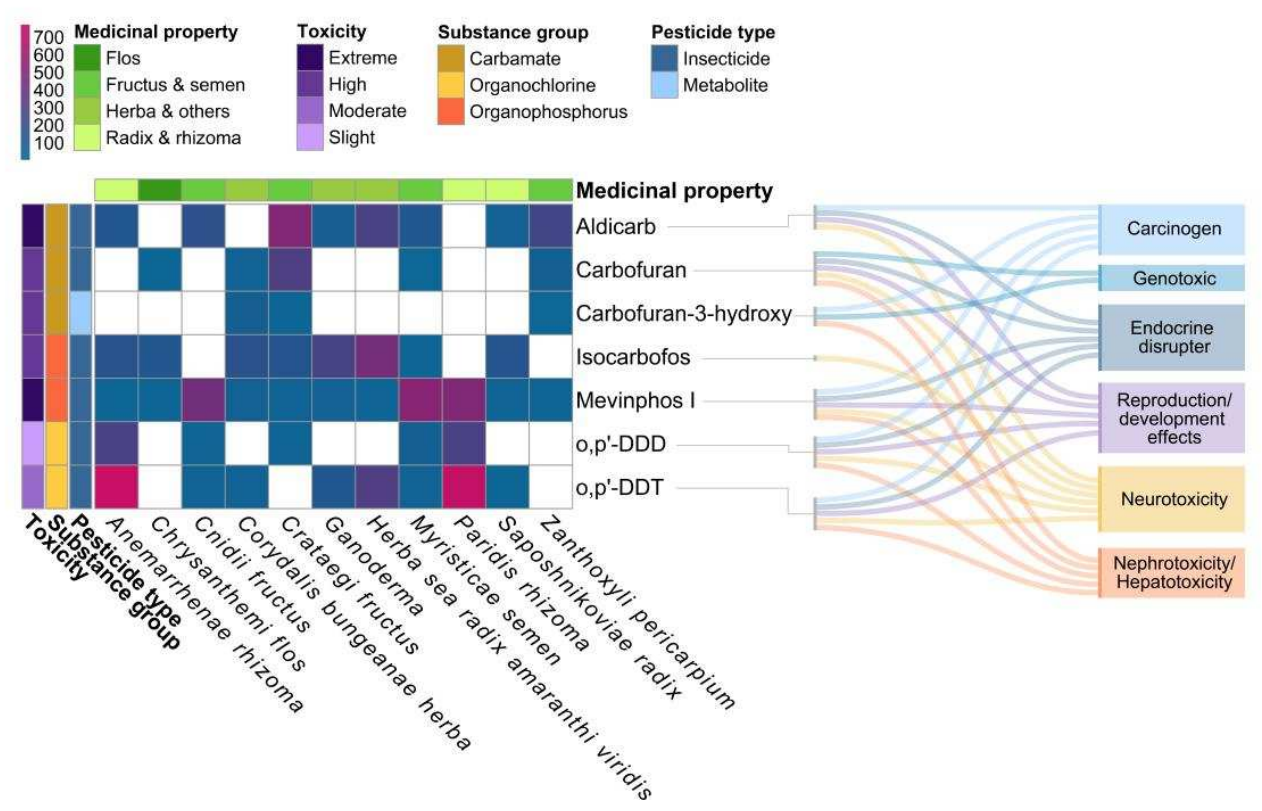

Fig.5: Heatmap of Impact Scores (mean $I_{\mathrm{i}, \mathrm{h}}$ ) of top seven risk-inducing (banned) pesticides in 11

\section{HM and their possible connections with health impacts}

Collecting $\mathrm{HQ}_{\mathrm{a}}>1$ and $\mathrm{HQ}_{\mathrm{c}}>1$ pesticides, and top ten pesticides with highest risk-ranking score $(\mathrm{S}=140)$ and Impact Score (mean $\mathrm{IS}_{\mathrm{i}, \mathrm{h}}$ ), ten pesticides were obtained. Since p,p'-DDT, leptophos, and methomyl were not detected in the $11 \mathrm{HM}$ included thus not shown here, totally seven banned pesticides were summarized above. Collecting $\mathrm{HQ}_{\mathrm{a}}>1$ and $\mathrm{HQ}_{\mathrm{c}}>1 \mathrm{HM}$, and top ten $\mathrm{HM}$ with highest risk index (RI) and Impact Score (mean $\mathrm{IS}_{\mathrm{h}}$ ), 11 risk-inducing herbal medicines appeared over once thus included here.

The possible health impacts of seven pesticides were derived from Pesticide Properties Data Base (PPDB) (University of Hertfordshire., 2019), European Food Safety Authority (EFSA) (EFSA, 2019), United States Environmental Protection Agency $(\mathrm{USEPA})^{47}$, Agency for Toxic Substances and Disease Registry (ATSDR) (Agency for Toxic Substances and Disease Registry, 2011), Toxicology Data Network from U.S. National Library of Medicine (TOXNET) (TOXNET, 2019), and Beyond Pesticides 
677

678

679

680

681

682

683

684

685

686

687

688

689

690

691

692

693

694

695

696

697

698

(Beyond Pesticides, 2018). Data showing positive, possible, mixed and ambiguous results are considered a possible connection,

while no data and negative results considered no connection.

In general, we propose four reasons why safety problems emerge from herbal medicinal products. First and most importantly, failure of surveillance and monitoring coupled with poor quality controls (Sahoo et al., 2010), this has resulted in non-standard and disproportionate use of pesticides and fertilizers and consequently contamination. This is in addition to adulteration, substitution, misidentification, incorrect processing, and inappropriate labeling and advertising (Garcia et al., 2012). Secondly, pesticide residues may not always be directly derived from their application but from accumulated residues in soil, wastewater irrigation, and aerial deposition (Vašíčcová et al., 2019). Both fructus and radix may assimilate pesticides from the soil through their roots (Han et al., 2017), which may be an explanation of why most pesticides were present in the radix and rhizome (Garcia et al., 2012). Thirdly, relatively safe pesticides with low toxicity and obvious effectiveness are neither sufficiently available nor well regulated, while highly and extremely toxic pesticides are easily accessible and inexpensive. Lastly, education and training institutions relating to safety, pesticide selection and, application for farmers is scarce.

Unlike intrinsic toxicity, contamination from extrinsic hazardous elements in HM can be regulated and controlled. Here we express a recommendation that regulatory efforts should be based on well-defined specifications that clearly standardize and classify HM cultivation, thus, ensuring better uniformity of quality, safety, and efficacy. A Chinese solution is herein proposed for regulating and establishing a universal standard for the production of HM. It includes four parts (Fig.6): (1) Good Agriculture 
699

700

701

702

703

704

705

706

707

708

709

710

711

712

713

714

715

Practice (GAP) \& Good Agricultural and Collection Practice (GACP) during cultivation, collection, and post-harvest operations for medicinal plants shall be implemented to ensure the quality of HM.

Standardized and regulated practices including ecological planting, wild nurturing, and bionic cultivation should be developed within formalized cultivation areas; (2) An appropriate unified standard with enforcement criteria regarding hazardous elements in HM must be established for HM production to control their safety and quality (Silva et al., 2019); (3) A legal framework regarding authentication and quality assessment, which incorporates three levels of certification, could be granted according to different levels of hazardous element contents such as pollution-free, green, and organic; (4) A comprehensive prediction, surveillance, and invigilation system should be created to predict, track, and check adverse events throughout the whole HM supply chain including shipping, importing, exporting, and maintenance of plant materials (Chen et al., 2015). An electronic database showcasing each step of HM production and its profile shall be constructed (Eisenberg et al., 2011). Therefore special focus on control and monitoring of risk-inducing pesticides including carbofuran, mevinphos, methomyl, isocarbofos, leptophos, and o,p'-DDT, as well as HMs Crataegi fructus, Lycii fructus, Zanthoxyli pericarpium, Corydalis bungeanae Herba, Myristicae semen, Cnidii fructus, Paridis rhizoma, Rhei radix et rhizoma, Radix chuanmingshinis violacei, and Coicis semen shall be needed, whose MRLs and dosages applied in clinics shall be refined based on our findings. (Table 1,2) 


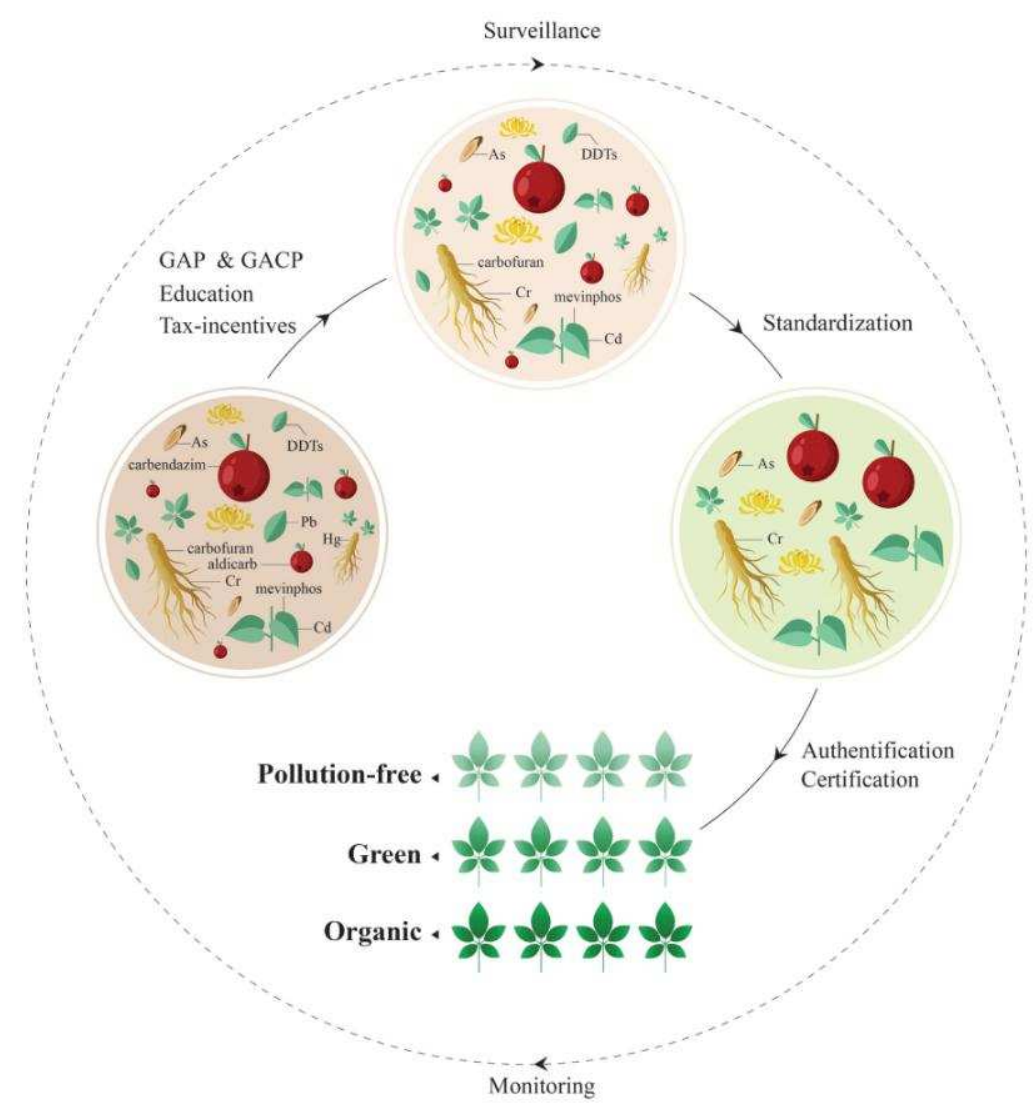


728

729

730

731

732

733

734

735

736

737

738

739

740

741

742

743

Further discussions, consultation, and harmonization, particularly relating to recommendations one, two, and three are desirable across a variety of geographic, cultural, and legislative landscapes in order to arrive at a meaningful and effective solution, since HMs are transported and consumed internationally. Though in this study the pesticide problem is confirmed, quantified, and further illuminated, future research around mapping the distribution and transmission of pesticides via their various vectors would be a welcome and valuable contribution to the field. Additional studies would deepen our understanding of the relationships between how pesticides are applied and the proportion from different vectors that make their way into HM. As little as $30 \%$ of sprayed pesticides reach their target (Zhu et al., 2019) and already complex intelligent systems are being designed for more targeted spraying (Asaei et al., 2019). The ecological influence of pesticides such as on the soil bacterial biome could also have an effect on intrinsic aspects of HM (Huang et al., 2018). If proposal four in particular here could be informed and refined by this kind of knowledge, there is further potential to optimize conditions to reduce intrinsic factors and direct future research areas to support a common goal of reducing pesticides and risk while increasing efficacy and safety.

We acknowledge that there are substantial challenges in implementing surveillance, tracking, and vigilance for $\mathrm{HM}$ on an international scale as outlined by proposal four However solutions for end-to-end technologies of full HM supply chain information management are already emerging (Heinrich et al., 2019). The apparent historical and current trend of good-will and increasing East-West co-operation in working together on HM quality standards is manifest recently with more comprehensive adoption of International Conference for Harmonization (ICH Q4) principles by the 
749

750

751

752

753

754

755

756

757

758

759

760

761

762

763

764

765

upcoming $11^{\text {th }}$ Edition Chinese Pharmacopoeia on one side, and European Directorate for the Quality of Medicines (EDQM) consulting with Chinese experts on the other. These factors collectively appear to support the credibility, feasibility, and timing of the proposals recommended here.

The potential limitations of our study merit considerations regarding pesticide profiles and risk assessments. As per the over-limit ratio, since the pesticide limit in HMs is not currently available, MRLs were derived according to the EP equation. For those pesticides in lack of ADIs, a default MRL of $0.01 \mathrm{mg} \cdot \mathrm{kg}^{-1}$ was defined. Without animal toxicological experiments, these ADIs and MRLs derived may not fully describe the realistic toxicity of pesticides, resulting in uncertainty of the over-limit situation of these eight pesticides. Of the 136 pesticides detected, their indicators including ARfD, ADI, NOEL, LOAEL, and $\mathrm{TD}_{50}$ were not completely available. Therefore, some pesticides were lost in the risk assessments. The dosages of each HMs were obtained from $\mathrm{CP}$, generally lower than the realistic dosage of herbal application in the clinic, leading to underestimated risks. For a realistic and comprehensive estimation, pesticide application data, and herbal processing factors (PF) should also be accounted for. We may assume that our interpretations of the pesticide contamination in HMs represent an over-estimate as only cultivated HMs were investigated, so it can't be extrapolated to predict the levels of pesticides in processed HMs and herbal supplements. Further researches will be warranted to determine the leaching behavior in HM preparations, general exposure scenarios, and assumed total daily intake of pesticides from HMs. Data on these aspects will refine the formal permissible limits of pesticides in HMs (Harris et al., 2011).

\section{Conclusions}


770

771

772

773

774

775

776

777

778

779

780

781

782

783

784

785

In conclusion, contamination from pesticide residues in HM is extensive and severe. Over half herbal medicines contained pesticides over the EP limit, and $43 \%$ contained 35 varieties of banned pesticides, eight of which were over 500 times higher than the default Maximum Residue Limit. DDTs, carbofuran, and mevinphos were confirmed as the most risk-inducing pesticides by three different risk assessments. Herbal medicines with fructus \& semen applied posed most serious risks, while pesticides were the most widely detected in radix \& rhizoma. Pesticides Aldicarb, carbofuran, isocarbofos, mevinphos, and DDTs, with above-threshold concentrations contained in herbal medicines, could potentially induce carcinogenic, genotoxic, endocrine disrupting, reproductive, development, neurotoxic, nephrontoxic, and heaptoxic pathologies, causing inreversible health disorders. It warrants further attention than previously given in light of its significant potential hazard to public health. Extrinsic contamination may be the neglected culprit in the adverse effects of HM. A globalized and regulated solution for surveilling, monitoring, and certifying the HM supply chain is proposed here with a goal of improved global health.

\section{Supplementary information}

Appendix which include the following tables and figures:

Table S1.1: List of 1000 herbal medicine samples for LC-MS

Table S1.2: List of 771 herbal medicine samples for GC-MS

Table S2.1: List of 136 pesticides and relative indicators

Table S2.2: Standard curve and R2 of 98 pesticides by LC-MS

Table S2.3: Standard curve and R2 of 44 pesticides by GC-MS

Table S2.4: Ion trace and retention time of 98 pesticides by LC-MS 
792

793

794

795

796

797

798

799

800

801

802

803

804

805

806

807

808

809

810

811

812

Table S2.5: Ion trace and retention time of 44 pesticides by GC-MS

Table S3: Eight ADI-vacant pesticides and 35 banned pesticides of MRLs calculated according to EP equation

Table S4: Weighting system of risk-ranking score of pesticides in herbal medicines

Table S5: Detection rate and over-limit ratio of pesticides, and detection rate of banned pesticides

Table S6: Number of detected pesticides, of over-limit pesticides and of banned pesticides in single herbal medicine

Table S7.1: Detection frequency of different numbers of pesticides in five medicinal properties by LC-MS \& GC-MS

Table S7.2: Detection frequency of six pesticide types in five medicinal properties by LC-MS \& GC-MS

Figure S1: Highest ranking pesticides and herbal medicines and proportion of risk-ranking scores

Figure S2: Violin plot of patient health impact assessment of pesticides in herbal medicines

Figure S3: Heatmap of risk-ranking scores (S) of 136 pesticides in 182 herbal medicines

Figure S4: Heatmap of impact score (mean ISi,h) of 136 pesticides in 182 herbal medicines

Figure S5: Comparative patient Impact Score (ISh) and Risk Index (RI) of 182 herbal medicines

\section{Abbreviations}

HM: herbal medicines

LC/MS-MS: liquid chromatography-tandem mass spectrometry

GC/MS-MS: gas chromatography-tandem mass spectrometry

SRM: selected reaction monitoring 
814

815

816

817

818

819

820

821

822

823

824

825

826

827

828

829

830

831

832

833

834

835
LOD: limit of detection

LOQ: limit of quantitation

EP: European Pharmacopoeia

MRL: Maximum Residue Limit

DDT: dichlorobiphenyl trichloroethane

HQ: hazard quotient

HI: hazard index

S: risk-ranking score

RI: Risk Index

IS: impact scores

DALY: disability-adjusted life years

ADI: Acceptable Daily Intake

ARfD: Acute Reference Dose

EU: European Union

US: United States

$\mathrm{DT}_{50}$ : half-life of degradation time

CNS: Central Nervous System

POPs: Persistent Organic Pollutants

GAP: Good Agriculture Practice

GACP: Good Agricultural and Collection Practice

ICH: International Conference for Harmonization

EDQM: European Directorate for the Quality of Medicines 
838 All authors declare no competing interests.

\section{Acknowledgements}

This study was founded by the Study on the Key Safety Validation Elements of Chinese Medicine in

842 International Trade, International Science \& Technology Cooperation Program, PR China

843 (2015DFM30030) and the OLCA-Pest Project, financially supported by ADEME, Denmark $844 \quad(17-03-\mathrm{C} 0025)$

\section{References}

847

Abou-Arab AAK, Abou-Donia MA. Pesticide residues in some Egyptian spices and medicinal plants as affected by processing. Food Chem 2001; 72: 439-45. DOI:10.1016/s0308-8146(00)00254-5.

Agency for Toxic Substances and Disease Registry. Health Effects of Exposure to Substances and Carcinogens. 2011. https://www.atsdr.cdc.gov/substances/ToxOrganSystems.asp (accessed May 21, 2018).

Adams RE, Brickel JA, Bhat VS. Chemical-specific maximum allowable levels for pesticide residues in dietary supplements. Food Chem Toxicol 2018; 123: 511-9. DOI:10.1016/j.fct.2018.11.045.

Adusei-Mensah F, Henneh IT, Ekor M. Pesticide Residue and Health Risk Analysis of Six Commonly Used Herbal Medicinal Products in Kumasi, Ghana. Texila International Journal of Public Health 2018; 6: 1-10. DOI: 10.21522/TIJPH.2013.06.03.Art018.

Asaei H, Jafari A, Loghavi M. Site-specific orchard sprayer equipped with machine vision for chemical usage management.

Comput Electron Agr 2019; 162: 431-9. DOI:10.1016/j.compag.2019.04.040. 
858

Broe D, Marc E. Chinese herbs nephropathy and Balkan endemic nephropathy: toward a single entity, aristolochic acid nephropathy. Kidney Int 2012; 81: 513-15. DOI: 10.1038/ki.2011.428.

Buffington EJ, McDonald SK. Banned and Severely Restricted Pesticides. https://webdoc.agsci.colostate.edu/cepep/FactSheets/141BannedPesticides.pdf. (accessed June 12, 2018).

Bhandari G, Zomer P, Atreya K, Mol HGJ, Yang X, Geissen V. Pesticide residues in Nepalese vegetables and potential health risks. Environ Res 2019; 172: 511-21. DOI:10.1016/j.envres.2019.03.002.

Bhanti M, Taneja A. Contamination of vegetables of different seasons with organophosphorous pesticides and related health risk assessment in northern India. Chemosphere 2007; 69: 63-68.

Beyond Pesticides. Gateway on Pesticide Hazards and Safe Pest Management. https://beyondpesticides.org/resources/pesticide-gateway?joiner=AND\#searchstart(accessed Jun 23, 2018)

Bilthoven, The Netherlands: National Institute for Health and the Environment, 2001. https://www.rivm.nl/bibliotheek/rapporten/601516005.pdf. (accessed October 11, 2018).

Beijing Xilinbuke Network Technology Co., Ltd. Chemical book. 2016. https://www.chemicalbook.com/Search.aspx? accessed August 11, 2018.

China Food Drug Administration. GB 2763-2016. National Food Safety Standard-Maximum residue limits for pesticides in food. Beijing, China: China Food Drug Administration; 2016.

Chou WC, Tsai WR, Chang HH, Lu SY, Lin KF, Lin P. Prioritization of pesticides in crops with a semi-quantitative risk ranking method for Taiwan postmarket monitoring program. J Food Drug Anal 2019; 27: 347-54. DOI:10.1016/j.jfda.2018.06.009.

Cherta L, Portolés T, Beltran J, Pitarch E, Mol JG, Hernández F. Application of gas chromatography-(triple quadrupole) mass 
880

881

882

883

884

885

886

887

888

889

890

891

892

893

894

895

896

897

898

899

900

spectrometry with atmospheric pressure chemical ionization for the determination of multiclass pesticides in fruits and vegetables.

J Chromatogr A 2013; 1314: 224-40. DOI: 10.1016/j.chroma.2013.09.029.

Colorado Environmental and Pesticide Education Program, Colorado State University. Colorado ChemSweep Pesticide Waste

Collection Program. http://www.cepep.colostate.edu/Disposal/Chemsweep\%20brochure.pdf. (accessed June 12, 2018).

Chen ZL, Dong FS, Xu J, Liu XG, Zheng YQ. Management of pesticide residues in China. J Integr Agr 2015; 14: 2319-27.

DOI:10.1016/S2095-3119(15)61110-8.

Debelle FD, Vanherweghem JL, Nortier JL. Aristolochic acid nephropathy: a worldwide problem. Kidney Int 2008; 74: 158-69. DOI:10.1038/ki.2008.129.

European

Commission

EU

Pesticide

Database.

https://ec.europa.eu/food/plant/pesticides/eu-pesticides-database/public/?event=activesubstance.selection\&language=EN.

Updated April 7, 2016. (accessed August 20, 2018).

European Commission. Guide to regulation (EC) of the European Parliament and of the Council No. 304/2003 concerning the $\begin{array}{llll}\text { export and } & \text { of } & \text { chemicals. }\end{array}$ http://www.chemicals.moew.government.bg/chemical/site/File/import\%20export/EDEXIM_GUIDE.pdf. (accessed July 13, 2018).

European Commission. SANCO Document 12495/2011 on Method Validation and Quality Control Procedures for Pesticide

Residues Analysis in Food and Feed. $\square$ http:/www.europa.eu.int/comm/food http:/www.crl-pesticides.eu. (accessed August 20, 2018).

European Directorate for the Quality Control of Medicines. Europen pharmacopoeia (8.0 $0^{\text {th }}$ edition). Strasbourg: European Directorate for the Quality of Medicines \& Health Care (EDQM) Council of Europe, 2014.

Eisenberg DM, Harris ES, Littlefield BA, et al. Developing a library of authenticated Traditional Chinese Medicinal (TCM) plants for systematic biological evaluation--rationale, methods and preliminary results from a Sino-American collaboration. 
902

903

904

905

906

907

908

909

910

911

912

913

914

915

916

917

918

919

920

921

922

Fitoterapia 2011; 82: 17-33. DOI: 10.1016/j.fitote.2010.11.017.

European Food Safety Authority. Chemical hazards data - OpenFoodTox. http://www.efsa.europa.eu/en/data/chemical-hazards-data (accessed May 19, 2019).

European Food Safety Authority. International Frameworks Dealing with Human Risk Assessment of Combined Exposure to Multiple Chemicals. EFSA Journal 2013; published July 12. DOI:10.2903/j.efsa.2013.3313.

FAO/WHO. Appendix F Approximate relation of parts per million in the diet to $\mathrm{mg} / \mathrm{kg}$ of body weight per day, Food additives Guidelines for the preparation of toxicological working papers for the Joint FAO/WHO Expert Committee on Food Additives.

Geneva, Switzerland: Joint FAO/WHO Expert Committee on Food Additives; 2000.

Fantke P, Friedrich R, Jolliet O. Health impact and damage cost assessment of pesticides in Europe. Environ Int 2012; 49: 9-17. DOI:10.1016/j.envint.2012.08.001.

Fantke P, Juraske R, Antón A, Friedrich R, Jolliet O. Dynamic multicrop model to characterize impacts of pesticides in food. Integr Environ Assess Manag. 2011; 45(20):8842-8849. doi:10.1021/es201989d.

Foodmate. net. http://db.foodmate.net/pesticide/search nyxz.php. Updated 2012. (accessed June 2, 2019).

Frischknecht R, Fantke P, Tschümperlin L, et al. Global guidance on environmental life cycle impact assessment indicators: progress and case study. Int J Life Cycle Ass 2016; 21: 429-42. DOI:10.1007/s11367-015-1025-1

Garcia FP, Ascencio SYC, Oyarzun JCG, Hernandez AC, Alavarado PV. Pesticides: classification, uses and toxicity. Measures of exposure and genotoxic risks. J $\quad$ Res $\quad$ Environ $\quad$ Sci $\quad$ Toxicol 2012; 1: $279-93$. (https://pdfs.semanticscholar.org/225e/6c4ebada0757fc67ef46d06a2347dec00335.pdf).

Harris ESJ, Cao S, Littlefield BA, et al. Heavy metal and pesticide content in commonly prescribed individual raw Chinese Herbal Medicines. Sci Total Environ, 2011, 409: 4297-4305.

Hajšlová J, Zrostlíková J. Matrix effects in (ultra)trace analysis of pesticide residues in food and biotic matrices. J Chromatogr A 2003; 1000: 181-97. DOI:10.1016/S0021-9673(03)00539-9. 
924

925

926

927

928

929

930

931

932

933

934

935

936

937

938

939

940

941

942

943

944

945

Huijbregts MA, Rombouts LJ, Ragas AM, van de Meent D. Human-Toxicological Effect and Damage Factors of Carcinogenic and Noncarcinogenic Chemicals for Life Cycle Impact Assessment. Integr Environ Asses 2005; 1: 181-244. DOI:10.1897/2004-007R.1.

Heinrich M, Scotti F, Booker A, Fitzgerald M, Kum KY, Löbel K. Unblocking high-value botanical value chains: Is there a role for blockchain systems?. Front Pharmacol 2019. DOI:10.3389/fphar.2019.00396.

Huang WJ, Long CL, Lam E. Roles of plant-associated microbiota in traditional herbal medicine. Trends Plant Sci 2018; 23: 559-62. DOI:10.1016/j.tplants.2018.05.003.

Han YH, Mo RH, Yuan XY, et al. Pesticide residues in nut-planted soils of China and their relationship between nut/soil. Chemosphere 2017; 180: 42-7. DOI:10.1016/j.chemosphere.2017.03.138.

Kumari D, John S. Health risk assessment of pesticide residues in fruits and vegetables from farms and markets of Western Indian Himalayan region. Chemosphere 2019; 224: 162-67. DOI:10.1016/j.chemosphere.2019.02.091.

Licensing and Regulatory Affairs. Department Banned Pesticide Active Ingredient List. https://komornlaw.com/wp-content/uploads/2018/04/Department_Banned_Pesticide_Active_Ingredient_List_620039 7.pdf.

Published April 9, 2018. (accessed August 19, 2018).

Lord, G. M., Tagore, R., Cook, T., Gower, P., \& Pusey, C. D. Nephropathy caused by Chinese herbs in the UK. Lancet, 1999; 354: 481-482.

Li ZX, Nie JY, Yan Z, et al. A monitoring survey and dietary risk assessment for pesticide residues on peaches in China. Regul Toxicol Phar 2018; 97: 152-62. DOI:10.1016/j.yrtph.2018.06.007.

Ministry of Agriculture and Rural Affairs of the People's Republic of China. List of banned pesticides. https://wenku.baidu.com/view/95318576c77da26924c5b0c5.html. (accessed July 5, 2018).

Ministry of Food and Drug Safety. Pesticide MRLs in Agricultural Commodities. South Korea: Residues and Contaminants Standard Division, Food Standards Planning Office, Ministry of Food and Drug Safety; 2019. 
946

$\mathrm{Ng}$ AWT, Poon SL, Huang MN, et al. Aristolochic acids and their derivatives are widely implicated in liver cancers in taiwan and throughout Asia. Science Transl Med 2017; 9: eaan6446. DOI: 10.1126/scitranslmed.aan6446.

Nortier JL, Martinez MC, Schmeiser HH, et al. Urothelial carcinoma associated with the use of a Chinese herb (Aristolochia fangchi). N Engl J Med 2000; 342: 1686-92. DOI:10.1056/NEJM200006083422301.

Nie JY, Li ZX, Liu CD, et al. Risk assessment of pesticide residues in apples. Sci Agric Sin 2014; 47: $3655-67$. DOI:10.3864/j.issn.0578-1752.2014.18.013.

National Pharmacopoeia Commission. Pharmacopoeia of the People's Republic of China, Part IV. Beijing, China: China Medical Science Press; 2015.

Nanjing University of Chinese Medicine. Encyclopedia of Chinese Herbal Medicine. Shanghai, China: Shanghai Scientific \&

Technical Publishers; 2006.

Pesticide Action Network, North America. PAN Pesticide Database - Chemicals. http://www.pesticideinfo.org. accessed August

$11,2018$.

Pesticide Action Network Europe. What substances are banned and authorised in the EU market?. https://www.pan-europe.info/old/Archive/About\%20pesticides/Banned\%20and\%20authorised.htm. Updated August 4, 2006. (accessed June 16, 2018).

Pytlakowska K, Kita A, Janoska P, Połowniak M, Kozik V. Multi-element analysis of mineral and trace elements in medicinal herbs and their infusions. Food Chem 2012, 135: 494-01.

Rosenbaum RK, Huijbregts MAJ, Henderson AD, et al. USEtox human exposure and toxicity factors for comparative assessment of toxic emissions in life cycle analysis: sensitivity to key chemical properties. Int J Life Cycle Ass 2011; 16: 710-27. DOI:10.1007/s11367-011-0316-4

Shah D, Benvenuti M, McCall E, Joshi S, Burgess JA. LC-MS/MS Analysis of Pesticide Residues in Rice and Unexpected Detection of Residues in an Organic Rice Sample. 2015. https://www.waters.com/webassets/cms/library/docs/720005285en.pdf (accessed Oct 16, 2018). 
The Commission of the European Communities. Commission Regulation (EC) No 777/2006 of 23 May 2006 amending Annex I

The Office for Official Publications of the European Communities. Council Directive 79/117/EEC prohibiting the placing on the market and use of plant protection products containing certain active substances. 
991

992

993

994

995

996

997

998

999

1000

1001

1002

1003

1004

1005

1006

1007

1008

1009

1010

1011

1012

of the Coucil of 28 January 2003 concerning the export and import of dangerous chemicals (Text with EEA relevance).

https://eur-lex.europa.eu/LexUriServ/LexUriServ.do?uri=CONSLEG:2003R0304:20071214:EN:PDF. (accessed July 13, 2018).

The European Parliament and the Council of the European Union. Regulation (EC) No 850/2004 of the European Parliament and

of the Council of 29 April 2004 on persistent organic pollutants and amending Directive 79/117/EEC.

https://eur-lex.europa.eu/LexUriServ/LexUriServ.do?uri=OJ:L:2004:158:0007:0049:EN:PDF. (accessed July 13, 2018).

The R Core Team. R: A Language and Environment for Statistical Computing. R Foundation for Statistical Computing.

https://www.R-project.org/. (accessed October 11, 2019).

Tripathy V, Basak BB, Varghese TS, Saha A. Residues and contaminants in medicinal herbs-A review. Phytochem Lett 2015; 14:

67-78. DOI:10.1016/j.phytol.2015.09.003.

University of Hertfordshire. Pesticide Properties DataBase (PPDB). 2019. https://sitem.herts.ac.uk/aeru/ppdb/en/atoz.htm\#A (accessed May 12, 2019).

United States Environmental Protection Agency. 875 Thousand Chemicals. 2019. https://comptox.epa.gov/dashboard (accessed May 21, 2018).

United States Environmental Protection Agency. Guidance on Cumulative Risk Assessment of Pesticide Chemicals That Have a Common Mechanism

of

Toxicity.

2002.

https://www.epa.gov/pesticide-science-and-assessing-pesticide-risks/guidance-cumulative-risk-assessment-pesticide (accessed

Feb 21, 2019).

U.S.Environmental Protection Agency. UN PIC \& $\quad$ U.S.Pic-Nominated Pesticides List. 2003. http://www.pic.int/Countries/CountryProfile/tabid/1087/language/en-US/Default.aspx. (accessed June 12, 2018).

U.S. Food \& Drug Administration. FDA/CVM GFI No. 3, General Principles for Evaluating the Safety of Compounds Used in Food-Producing

Animals.

http://www.fda.gov/downloads/AnimalVeterinary/GuidanceComplianceEnforcement/GuidanceforIndustry/UCM052180.pdf.

Published June, 2018. (accessed May 16, 2019). 
1014

1015

1016

1017

1018

1019

1020

1021

1022

1023

1024

1025

1026

1027

1028

1029

1030

1031

1032

1033

1034

1035

VRC. Annual report on surveillance for veterinary residues in food in the UK. UK: Veterinary Residues Committee, 2007.

https://assets.publishing.service.gov.uk/government/uploads/system/uploads/attachment_data/file/243614/9780108507656.pdf

Vania C. Management of change in health care organizations and human resource role. Eur J Radiol. 2000; 33(1): 8-13.

doi:10.1016/S0720-048X(99)00130-8.

Vašíčková J, Hvězdová M, Kosubová P, Hofman J, Hofman J. Ecological risk assessment of pesticide residues in arable soils of

the Czech Republic. Chemosphere 2019; 216: 479-87. DOI:10.1016/j.chemosphere.2018.10.158.

Vermeire T, Pieters M, Rennen M, Bos P. Probabalistic assessment factors for human health risk assessment—A practical guide.

World Health Organization. The WHO Recommended Classification of Pesticides by Hazard And Guidelines to Classification

2009. https://www.who.int/foodsafety/publications/classification-pesticides/en/. Published 2010. accessed July 2, 2019.

Wang Y, Liu A, Li J, et al. The Survey of Traditional Chinese Medicine Consumption among Adult Residents in 11 Provinces and

Cities. Chinese Pharmaceutical Affairs 2017; 31: 666-72. DOI:10.16153/j.1002-7777.2017.06.016.

$\begin{array}{lllll}\text { Waters } & \text { Corporation. } & \text { DisQuE }\end{array}$ https://www.waters.com/webassets/cms/library/docs/720003048en.pdf (accessed July 16, 2018).

WHO. WHO Traditional Medicine Strategy 2014-2023. Geneva: World Health Organization, 2013. https://www.who.int/medicines/publications/traditional/trm_strategy14_23/en/ (accessed Aug 22, 2019).

Xiao JJ, Xu X, Wang F, et al. Analysis of exposure to pesticide residues from Traditional Chinese Medicine consumption in China. J Hazard Mater 2019; 365: 857-67. DOI:10.1016/j.jhazmat.2018.11.075.

Yang HY, Chen PC, Wang JD. Chinese herbs containing aristolochic acid associated with renal failure and urothelial carcinoma: a review from epidemiologic observations to causal inference. Biomed Res Int 2014; 2014: 569325. DOI: 10.1155/2014/569325.

Zhu H, Derksen R, Guler H, Krause C, Ozkan H. Foliar deposition and offtarget loss with different spray techniques in nursery applications. Trans ASABE 2006; 49: 325-34. DOI: 10.1016/j.compag.2019.04.040

Zuo TT, Wang Y, Zhang L, et al. Guideline of risk assessment of exogenous harmful residues in traditional Chinese medicines. 
Chin J Pharm Anal 2019; 39: 1902-07. DOI:10.16155/j.0254-1793.2019.10.20.

1037

Zuin V G., Vilegas J H.Y. Pesticide residues in medicinal plants and phytomedicines. Phytother Res 2000; 14:73-88. 


\section{Highlight}

Extrinsic contamination in herbal medicines is severe with extremely toxic and banned pesticides detected.

DDTs, carbofuran, and mevinphos were confirmed as the most risk-inducing pesticides.

Crataegi fructus posed most serious risks, while pesticides were most widely detected in radix \& rhizoma.

A globalized and regulated solution for surveilling, monitoring, and certifying the HM supply chain is in urgent need. 


\section{Declaration of interests}

$\checkmark$ The authors declare that they have no known competing financial interests or personal relationships that could have appeared to influence the work reported in this paper.

$\square$ The authors declare the following financial interests/personal relationships which may be considered as potential competing interests:

All authors declare no competing interests. 